\title{
Closed-form solution of visual-inertial structure from motion
}

\author{
Agostino Martinelli
}

Received: date / Accepted: date

\begin{abstract}
This paper investigates the visual-inertial structure from motion problem. A simple closed form solution to this problem is introduced. Special attention is devoted to identify the conditions under which the problem has a finite number of solutions. Specifically, it is shown that the problem can have a unique solution, two distinct solutions and infinite solutions depending on the trajectory, on the number of point-features and on their layout and on the number of camera images. The investigation is also performed in the case when the inertial data are biased, showing that, in this latter case, more images and more restrictive conditions on the trajectory are required for the problem resolvability.
\end{abstract}

Keywords Sensor Fusion - Structure from Motion · Inertial Sensors · Robotics

\section{Introduction}

The structure from motion problem (SfM) consists of determining the three-dimensional structure of the scene by using the measurements provided by one or more sensors over time (e.g. vision sensors, ego-motion sensors, range sensors). In the case of visual measurements only, the SfM problem has been solved up to a scale $[4,5,11,16,20]$ and a closed form solution has also been derived $[11,16,20]$, allowing the determination of the three-dimensional structure of the scene, without the need for any prior knowledge.

The case of inertial and visual measurements, i.e., the visual-inertial structure from motion problem (from

\footnotetext{
Agostino Martinelli

Emotion INRIA Rhone Alpes

Tel.: +33-476-615557

E-mail: agostino.martinelli@inria.fr
}

now on the Vi-SfM problem), has particular interest and has been investigated by many disciplines, both in the framework of computer science $[3,13,14,17,21]$ and in the framework of neuroscience (e.g., $[2,6,9])$. Prior work has answered the question of which are the observable modes, i.e. the states that can be determined by fusing visual and inertial measurements $[3,13,14$, 17]. Specifically, it has been shown that the velocity, the absolute scale, the gravity vector in the local frame and the bias-vectors which affect the inertial measurements, are observable modes. On the other hand, the problem of determining these observable modes is not fully solved.

The majority of the approaches so far introduced, perform the fusion of vision and inertial sensors by filter-based algorithms. In [1], these sensors are used to perform egomotion estimation. The sensor fusion is obtained by an Extended Kalman Filter $(E K F)$ and by an Unscented Kalman Filter $(U K F)$. The approach proposed in [10] extends the previous one by also estimating the structure of the environment where the motion occurs. Also, in [22] an EKF has been adopted. In this case, the proposed algorithm estimates a state containing the robot speed, position and attitude, together with the inertial sensor biases and the location of the features of interest. In the framework of airbone SfM, an EKF has been adopted in [15] to solve the Vi-SfM problem. It was observed that any inconsistent attitude update severely affects any SfM solution. The authors proposed to separate attitude update from position and velocity update. Alternatively, they proposed to use additional velocity observations, such as air velocity observation. Very recently, in the frame work of micro aerial robotics, flight stabilization and fully autonomous navigation have been achieved by using monocular vision and inertial sensors as the only 
exteroceptive sensors. Also in this case, the sensor fusion was carried out by a filter based algorithm $[23,24]$. There are very few methods able to perform the fusion of image and inertial measurements without a filterbased approach. One algorithm of this type has been suggested in [21]. This algorithm is a batch method which performs SfM from image and inertial measurements. Specifically, it minimizes a cost function by using the Leven-Marquardt algorithm. This minimization process starts by initializing the velocities, the gravity and the biases to zero.

When using a linear estimator (e.g. an $E K F$ ), or an optimization method in order to minimize a suitable cost function, an important issue which arises is the initialization problem. Indeed, because of the system non-linearities, lack of a precise initialization can irreparably damage the entire estimation process. This important limitation would be eliminated by introducing a deterministic solution, i.e., by analytically expressing all the observable modes in terms of the measurements provided by the sensors during a short timeinterval. Closed form solutions have been introduced very recently in [17]. Specifically, in [17] an observability analysis allowed us to quantify the information resulting when combining visual and inertial measurements. This allowed us to analytically derive the observable modes. Then, starting from the differential equations which characterize a generic $3 D$-motion and from the analytical expression of the visual observations, closed form expressions of the observable modes in terms of the sensor measurements were derived. On the other hand, these derivations did not allow us to detect the conditions under which the Vi-SfM can be solved. This important issue was very marginally investigated in [17]. Specifically, the observability analysis carried out in [17] only allowed us to detect a very limited number of singular cases where the sensor information does not allow us to determine the observable modes.

Here we derive a new simple and intuitive closed solution to the Vi-SfM problem. Compared with the solutions proposed in [17], this new solution allows us to investigate the intrinsic properties of the Vi-SfM problem and to identify the conditions under which the problem can be solved in closed form. In particular, these conditions regard the trajectory, the number of pointfeatures and their layout and the number of monocular images where the same point-features are seen. Additionally, minimal cases have been fully investigated, i.e., necessary and sufficient conditions on the trajectory and on the feature layout have been provided for the cases when the number of features and the number of camera images is the minimum required for the Vi-SfM problem resolvability.
All the theoretical results derived in this paper are obtained under the assumption of noiseless visual and inertial measurements. Additionally, the measurements provided by the gyroscopes are assumed to be unbiased (only the case of a constant bias on the accelerometers is considered). Finally, the theoretical analysis assumes that all the sensors share the same reference frame (in other words, the transformation between visual and inertial sensors is a priori known). Very recently, a closed form determination of this transformation has been suggested [7]. On the other hand, Monte Carlo simulations have also been performed by relaxing all these assumptions.

The paper is organized as follows. The system is defined in section 2 . In section 3 the Vi-SfM problem is reduced to a polynomial equation system, whose resolvability is investigated in section 4 , both in the unbiased (4.1) case and in the case of biased accelerometer measurements (4.2). All the possible cases are then summarized in the first part of section 5. In section 5.2 some results obtained by performing Monte Carlo simulations are also provided. Specifically, the assumptions made in the theoretical analysis are relaxed in order to generate the sensor measurements and the closed form solution is used in conjunction with a filtering approach in order to show its benefit. Finally, concluding remarks are provided in section 6 .

\section{The Considered System}

We consider a system (from now on we call it the platform) consisting of a monocular camera and an Inertial Measurement Unit $(I M U)$. The IMU consists of three orthogonal accelerometers and three orthogonal gyrometers. We introduce a global frame in order to characterize the motion of the platform moving in a $3 D$ environment. Its $z$-axis points vertically upwards. As we will see, for the next derivation we do not need to better define this global frame. We will adopt lower-case letters to denote vectors in this frame (e.g. the gravity is $\boldsymbol{g}=[0,0,-g]^{T}$, where $\left.g \simeq 9.8 \mathrm{~ms}^{-2}\right)$. We assume that the transformations among the camera frame and the IMU frame are known (we assume that the platform frame coincides with the camera frame and we call it the local frame). We will adopt upper-case letters to denote vectors in this frame. Since this local frame is time dependent, we adopt the following notation: $\boldsymbol{W}_{t}(\tau)$ will be the vector with global coordinates $\boldsymbol{w}(\tau)$ in the local frame at time $t$. Additionally, we will denote with $C_{t_{1}}^{t_{2}}$ the matrix which characterizes the rotation occurred during the time interval $\left(t_{1}, t_{2}\right)$ and with $C_{t_{2}}^{t_{1}}$ its inverse (i.e., $\left.\left(C_{t_{1}}^{t_{2}}\right)^{-1}=C_{t_{2}}^{t_{1}}\right)$. Let us refer 
to vectors which are independent of the origin of the reference frame (e.g., speed, acceleration, etc.). For these vectors we have: $\boldsymbol{W}_{t_{1}}(\tau)=C_{t_{1}}^{t_{2}} \boldsymbol{W}_{t_{2}}(\tau)$. Finally, $C^{t}$ will denote the rotation matrix between the global frame and the local frame at time $t$, i.e., $\boldsymbol{w}(\tau)=C^{t} \boldsymbol{W}_{t}(\tau)$.

The $I M U$ provides the platform angular speed and acceleration. Regarding the acceleration, the one perceived by the accelerometer $(\boldsymbol{A})$ is not simply the inertial acceleration $\left(\boldsymbol{A}^{\text {inertial }}\right)$. It also includes the gravitational acceleration $(\boldsymbol{G})$.

We assume that the camera is observing one or more point-features during the time interval $\left[T_{i n}, T_{f i n}\right]$. The platform and one of these observed features are displayed in fig 1 .

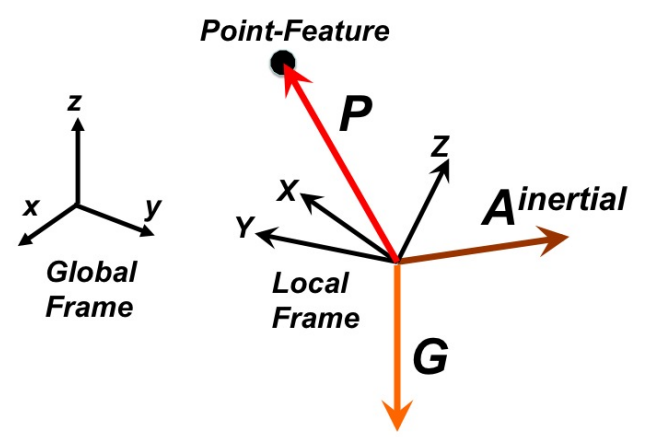

Fig. 1 Global and local frame with the point-feature position $(\boldsymbol{P})$, the platform acceleration $\left(\boldsymbol{A}^{\text {inertial }}\right)$ and the gravitational acceleration $(\boldsymbol{G})$.

\section{The closed form solution}

Prior work has answered the question of which are the observable modes, i.e. the states that can be determined by fusing visual and inertial measurements [3,13,14, 17]. The observable modes are: the platform velocity, the absolute scale, the gravity vector in the local frame and the bias-vectors which affect the inertial measurements. Note that the knowledge of the gravity in the local frame is equivalent to the knowledge of its magnitude together with the roll and pitch angle, i.e., the orientation of the platform with respect to the horizontal plane. Our goal is to express in closed-form all the observable modes at a given time $T_{i n}$ only in terms of the visual and inertial measurements obtained during the time interval $\left[T_{i n}, T_{f i n}\right]$.

The position of the platform $\boldsymbol{r}$ at any time $t \in$ $\left[T_{i n}, T_{\text {fin }}\right]$ satisfies the equation $\boldsymbol{r}(t)=\boldsymbol{r}\left(T_{i n}\right)+\boldsymbol{v}\left(T_{i n}\right) \Delta t+$ $\int_{T_{i n}}^{t} \int_{T_{i n}}^{\tau} \boldsymbol{a}(\xi) d \xi d \tau$. The last term contains a double in- tegral over time, which can be simplified in a single integral by integrating by parts. We obtain:

$\boldsymbol{r}(t)=\boldsymbol{r}\left(T_{i n}\right)+\boldsymbol{v}\left(T_{i n}\right) \Delta t+\int_{T_{i n}}^{t}(t-\tau) \boldsymbol{a}(\tau) d \tau$

where $\boldsymbol{v} \equiv \frac{d \boldsymbol{r}}{d t}, \boldsymbol{a} \equiv \frac{d \boldsymbol{v}}{d t}$ and $\Delta t \equiv t-T_{i n}$. The accelerometer does not provide the vector $\boldsymbol{a}(\tau)$. It provides the acceleration in the local frame and it also perceives the gravitational component. Additionally, its data are usually biased $[8,25]$, i.e., they are corrupted by a constant term $(\boldsymbol{B})^{1}$. In other words, the accelerometer provides the vector: $\boldsymbol{A}_{\tau}(\tau) \equiv \boldsymbol{A}_{\tau}^{\text {inertial }}(\tau)-\boldsymbol{G}_{\tau}+\boldsymbol{B}$. Note that the gravity comes with a minus since, when the platform does not accelerate (i.e. $\boldsymbol{A}_{\tau}^{\text {inertial }}(\tau)$ is zero), the accelerometer perceives an acceleration which is the same of an object accelerated upward in absence of gravity. Note also that the vector $\boldsymbol{G}_{\tau}$ depends on time only because the local frame can rotate.

We write equation (1) by highlighting the vector $\boldsymbol{A}_{\tau}(\tau)$ provided by the accelerometer:

$\boldsymbol{r}(t)=\boldsymbol{r}\left(T_{i n}\right)+\boldsymbol{v}\left(T_{i n}\right) \Delta t+\boldsymbol{g} \frac{\Delta t^{2}}{2}+C^{T_{i n}}\left[\boldsymbol{S}_{T_{i n}}(t)-\Gamma(t) \boldsymbol{B}\right]$

where:

$$
\begin{aligned}
& \boldsymbol{S}_{T_{i n}}(t) \equiv \int_{T_{i n}}^{t}(t-\tau) C_{T_{i n}}^{\tau} \boldsymbol{A}_{\tau}(\tau) d \tau \\
& \Gamma(t) \equiv \int_{T_{i n}}^{t}(t-\tau) C_{T_{i n}}^{\tau} d \tau
\end{aligned}
$$

The matrix $C_{T_{i n}}^{\tau}$ can be obtained from the angular speed during the interval $\left[T_{i n}, \tau\right]$ provided by the gyroscopes [8]. Hence, also the matrix $\Gamma(t)$ can be obtained by directly integrating the gyroscope data during the interval $\left[T_{i n}, t\right]$. Finally, the vector $\boldsymbol{S}_{T_{i n}}(t)$ can be obtained by integrating the data provided by the gyroscopes and the accelerometers delivered during the interval $\left[T_{i n}, t\right]$.

Let us suppose that $N$ point-features are observed, simultaneously. Let us denote their position in the physical world with $\boldsymbol{p}^{i}, i=1, \ldots, N$. According to our notation, $\boldsymbol{P}_{t}^{i}(t)$ will denote their position at time $t$ in the local frame at time $t$. We have:

1 Actually, the accelerometer bias slightly changes with time, i.e., it would be more appropriate to write $\boldsymbol{B}(\tau)$. However, as we will show in the next section, few camera images allow us to determine this component and we can assume that the bias is constant during the time interval needed to collect few camera images. 
$\boldsymbol{p}^{i}=\boldsymbol{r}(t)+C^{T_{i n}} C_{T_{i n}}^{t} \boldsymbol{P}_{t}^{i}(t)$

We write this equation at time $t=T_{\text {in }}$ obtaining:

$\boldsymbol{p}^{i}-\boldsymbol{r}\left(T_{i n}\right)=C^{T_{i n}} \boldsymbol{P}_{T_{i n}}^{i}\left(T_{i n}\right)$

By inserting the expression of $\boldsymbol{r}(t)$ provided in (2) into equation (3), by using (4) and by pre multiplying by the rotation matrix $\left(C^{T_{i n}}\right)^{-1}$ (we remind the reader that, according to our notation, $\boldsymbol{v}\left(T_{i n}\right)=C^{T_{i n}} \boldsymbol{V}_{T_{i n}}\left(T_{i n}\right)$ and $\boldsymbol{g}=C^{T_{i n}} \boldsymbol{G}_{T_{i n}}$ ) we finally obtain the following equation:

$$
\begin{gathered}
C_{T_{i n}}^{t} \boldsymbol{P}_{t}^{i}(t)=\boldsymbol{P}_{T_{i n}}^{i}\left(T_{i n}\right)-\boldsymbol{V}_{T_{i n}}\left(T_{i n}\right) \Delta t-\boldsymbol{G}_{T_{i n}} \frac{\Delta t^{2}}{2}+ \\
\Gamma(t) \boldsymbol{B}-\boldsymbol{S}_{T_{i n}}(t) ; \quad i=1,2, \ldots, N
\end{gathered}
$$

A single image provides the bearing angles of all the point-features in the local frame. In other words, an image taken at time $t$ provides all the vectors $\boldsymbol{P}_{t}^{i}(t)$ up to a scale. Since the data provided by the gyroscopes during the interval $\left(T_{i n}, T_{\text {fin }}\right)$ allow us to build the matrix $C_{T_{i n}}^{t}$, having the vectors $\boldsymbol{P}_{t}^{i}(t)$ up to a scale, allows us to also know the vectors $C_{T_{i n}}^{t} \boldsymbol{P}_{t}^{i}(t)$ up to a scale.

We assume that the camera provides $n_{i}$ images of the same $N$ point-features at the consecutive times: $t_{1}=T_{i n}<t_{2}<\ldots<t_{n_{i}}=T_{\text {fin }}$. From now on, for the sake of simplicity, we adopt the following notation:

$-\boldsymbol{P}_{j}^{i} \equiv C_{T_{i n}}^{t_{j}} \boldsymbol{P}_{t_{j}}^{i}\left(t_{j}\right), i=1,2, \ldots, N ; j=1,2, \ldots, n_{i}$

$-\boldsymbol{P}^{i} \equiv \boldsymbol{P}_{T_{i n}}^{i}\left(T_{i n}\right), i=1,2, \ldots, N$

- $\boldsymbol{V} \equiv \boldsymbol{V}_{T_{i n}}\left(T_{i n}\right)$

$-\boldsymbol{G} \equiv \boldsymbol{G}_{T_{i n}}$

$-\Gamma_{j} \equiv \Gamma\left(t_{j}\right), j=1,2, \ldots, n_{i}$

$-\boldsymbol{S}_{j} \equiv \boldsymbol{S}_{T_{i n}}\left(t_{j}\right), j=1,2, \ldots, n_{i}$

Additionally, we will denote with $\boldsymbol{\mu}_{j}^{i}$ the unit vector with the same direction of $\boldsymbol{P}_{j}^{i}$ and we introduce the unknowns $\lambda_{j}^{i}$ such that $\boldsymbol{P}_{j}^{i}=\lambda_{j}^{i} \boldsymbol{\mu}_{j}^{i}$. Finally, without loss of generality, we can set $T_{i n}=0$, i.e., $\Delta t=t$. Our sensors provide $\boldsymbol{\mu}_{j}^{i}$ and $\boldsymbol{S}_{j}$ for $i=1,2, \ldots, N$; $j=1,2, \ldots, n_{i}$. Equation (5) can be written as follows:

$$
\boldsymbol{P}^{i}-\boldsymbol{V} t_{j}-\boldsymbol{G} \frac{t_{j}^{2}}{2}+\Gamma_{j} \boldsymbol{B}-\lambda_{j}^{i} \boldsymbol{\mu}_{j}^{i}=\boldsymbol{S}_{j}
$$

The Vi-SfM problem is the determination of the vectors: $\boldsymbol{P}^{i},(i=1,2, \ldots, N), \boldsymbol{V}, \boldsymbol{G}$. In the case with biased accelerometer data, we also need to determine the vector $\boldsymbol{B}$. We can use the equations in (6) to determine these vectors. On the other hand, the use of (6) requires to also determine the quantities $\lambda_{j}^{i}$. By considering $j=1$ in (6), i.e. $t_{j}=t_{1}=T_{i n}=0$, we easily obtain: $\boldsymbol{P}^{i}=\lambda_{1}^{i} \boldsymbol{\mu}_{1}^{i}$. Then, we write the linear system in (6) as follows:

$\left[\begin{array}{c}-\boldsymbol{G} \frac{t_{j}^{2}}{2}-\boldsymbol{V} t_{j}+\Gamma_{j} \boldsymbol{B}+\lambda_{1}^{1} \boldsymbol{\mu}_{1}^{1}-\lambda_{j}^{1} \boldsymbol{\mu}_{j}^{1}=\boldsymbol{S}_{j} \\ \lambda_{1}^{1} \boldsymbol{\mu}_{1}^{1}-\lambda_{j}^{1} \boldsymbol{\mu}_{j}^{1}-\lambda_{1}^{i} \boldsymbol{\mu}_{1}^{i}+\lambda_{j}^{i} \boldsymbol{\mu}_{j}^{i}=0_{3}\end{array}\right.$

where $j=2, \ldots, n_{i}, i=2, \ldots, N$ and $0_{3}$ is the $3 \times 1$ zero vector. This linear system consists of $3\left(n_{i}-1\right) N$ equations in $N n_{i}+6$ unknowns (or $N n_{i}+9$ in the biased case). Let us define the two column vectors $\boldsymbol{X}$ and $\boldsymbol{S}$ :

$\boldsymbol{X} \equiv\left[\boldsymbol{G}^{T}, \boldsymbol{V}^{T}, \boldsymbol{B}^{T}, \lambda_{1}^{1}, \ldots, \lambda_{1}^{N}, \ldots, \lambda_{n_{i}}^{1}, \ldots, \lambda_{n_{i}}^{N}\right]^{T}$

(or

$\boldsymbol{X} \equiv\left[\boldsymbol{G}^{T}, \boldsymbol{V}^{T}, \lambda_{1}^{1}, \ldots, \lambda_{1}^{N}, \ldots, \lambda_{n_{i}}^{1}, \ldots, \lambda_{n_{i}}^{N}\right]^{T}$

in absence of bias), and

$\boldsymbol{S} \equiv\left[\boldsymbol{S}_{2}^{T}, 0_{3}, \ldots, 0_{3}, \boldsymbol{S}_{3}^{T}, 0_{3}, \ldots, 0_{3}, \ldots, \boldsymbol{S}_{n_{i}}^{T}, 0_{3}, \ldots, 0_{3}\right]^{T}$

and the matrix:

$\Xi \equiv$

$\left[\begin{array}{c|c|c|c|c|c|c|c|c|c|c|c}T_{2} & S_{2} & \Gamma_{2} & \boldsymbol{\mu}_{1}^{1} & 0_{3} & 0_{3} & -\boldsymbol{\mu}_{2}^{1} & 0_{3} & 0_{3} & 0_{3} & 0_{3} & 0_{3} \\ 0_{33} & 0_{33} & 0_{33} & \boldsymbol{\mu}_{1}^{1} & -\boldsymbol{\mu}_{1}^{2} & 0_{3} & -\boldsymbol{\mu}_{2}^{1} & \boldsymbol{\mu}_{2}^{2} & 0_{3} & 0_{3} & 0_{3} & 0_{3} \\ \ldots & \ldots & \ldots & \ldots & \ldots & \ldots & \ldots & \ldots & \ldots & \ldots & \ldots & \ldots \\ 0_{33} & 0_{33} & 0_{33} & \boldsymbol{\mu}_{1}^{1} & 0_{3} & -\boldsymbol{\mu}_{1}^{N} & -\boldsymbol{\mu}_{2}^{1} & 0_{3} & \boldsymbol{\mu}_{2}^{N} & 0_{3} & 0_{3} & 0_{3} \\ \ldots & \ldots & \ldots & \ldots & \ldots & \ldots & \ldots & \ldots & \ldots & \ldots & \ldots & \ldots \\ \ldots & \ldots & \ldots & \ldots & \ldots & \ldots & \ldots & \ldots & \ldots & \ldots & \ldots & \ldots \\ T_{n_{i}} & S_{n_{i}} & \Gamma_{n_{i}} & \boldsymbol{\mu}_{1}^{1} & 0_{3} & 0_{3} & 0_{3} & 0_{3} & 0_{3} & -\boldsymbol{\mu}_{n_{i}}^{1} & 0_{3} & 0_{3} \\ 0_{33} & 0_{33} & 0_{33} & \boldsymbol{\mu}_{1}^{1} & -\boldsymbol{\mu}_{1}^{2} & 0_{3} & 0_{3} & 0_{3} & 0_{3} & -\boldsymbol{\mu}_{n_{i}}^{1} & \boldsymbol{\mu}_{n_{i}}^{2} & 0_{3} \\ \ldots & \ldots & \ldots & \ldots & \ldots & \ldots & \ldots & \ldots & \ldots & \ldots & \ldots & \ldots \\ 0_{33} & 0_{33} & 0_{33} & \boldsymbol{\mu}_{1}^{1} & 0_{3} & -\boldsymbol{\mu}_{1}^{N} & 0_{3} & 0_{3} & 0_{3} & -\boldsymbol{\mu}_{n_{i}}^{1} & 0_{3} & \boldsymbol{\mu}_{n_{i}}^{N}\end{array}\right]$

where $T_{j} \equiv-\frac{t_{j}^{2}}{2} I_{3}, S_{j} \equiv-t_{j} I_{3}$ and $I_{3}$ is the identity $3 \times 3$ matrix; $0_{33}$ is the $3 \times 3$ zero matrix (note that the third set of columns disappear in absence of bias). The linear system in (7) can be written in the following compact format:

$\Xi X=S$

The sensor information is completely contained in the above linear system. Additionally, we assume that the magnitude of the gravitational acceleration is a priori known. This extra information is obtained by adding to our linear system the following quadratic equation: $|\boldsymbol{G}|=g$. By introducing the following $3 \times\left(N n_{i}+6\right)$ 
matrix (or $3 \times\left(N n_{i}+9\right)$ in the biased case), $\Pi \equiv$ $\left[I_{3}, 0_{3} \ldots 0_{3}\right]$, this quadratic constraint can be written in terms of $\boldsymbol{X}$ as follows:

$|\Pi \boldsymbol{X}|^{2}=g^{2}$

The Vi-SfM problem can be solved by finding the vector $\boldsymbol{X}$, which satisfies (9) and (10).

\section{Existence and number of distinct solutions}

We are interested in understanding how the existence and the number of solutions of the Vi-SfM problem depend on the motion, on the number of observed pointfeatures, on the point-features layout and on the number of camera images. The resolvability of the Vi-SfM problem can be investigated by computing the null space of the matrix $\Xi$ in $(8)$. Let us denote with $\mathcal{N}(\Xi)$ this space. The following theorem holds:

Theorem 1 (Number of Solutions) The Vi-SfM problem has a unique solution if and only if $\mathcal{N}(\Xi)$ is empty. It has two solutions, if and only if $\mathcal{N}(\Xi)$ has dimension 1 and, for any $\boldsymbol{n} \in \mathcal{N}(\Xi),|\Pi \boldsymbol{n}| \neq 0$. It has infinite solutions in all the other cases.

Proof : The first part of this theorem is a trivial consequence of the theory of linear systems. Indeed, the vector $\boldsymbol{X}$ can be uniquely obtained by inverting the matrix $\Xi$. Let us consider the case when the dimension of $\mathcal{N}(\Xi)$ is 1 . The linear system in (9) has infinite solutions with the following structure: $\boldsymbol{X}(\gamma)=\Xi^{*} \boldsymbol{S}+\gamma \boldsymbol{n}$, where $\Xi^{*}$ is a pseudoinverse of $\Xi, \boldsymbol{n}$ is a vector belonging to $\mathcal{N}(\Xi)$ and $\gamma$ is an unknown scalar value [19]. We use (10) to obtain $\gamma$. We have: $|\Pi \boldsymbol{X}(\gamma)|^{2}=g^{2}$, which is a second order polynomial equation in $\gamma$ if and only if $|\Pi \boldsymbol{n}| \neq 0$. Hence, we have two solutions for $\gamma$, $\gamma_{1}$ and $\gamma_{2}$, and two solutions for $\boldsymbol{X}, \boldsymbol{X}_{1} \equiv \boldsymbol{X}\left(\gamma_{1}\right)$ and $\boldsymbol{X}_{2} \equiv \boldsymbol{X}\left(\gamma_{2}\right)$. When $|\Pi \boldsymbol{n}|=0$ equation $|\Pi \boldsymbol{X}(\gamma)|^{2}=g^{2}$ is independent of $\gamma$. Hence, this equation is automatically satisfied, independently of $\gamma$. This means that the Vi-SfM problem has infinite solutions. However, it also means that the vector $\boldsymbol{G}$ can be uniquely determined.

The previous theorem allows us to obtain all the properties of the Vi-SfM problem by investigating the null space of $\Xi$. The dimension of this null space does not change by multiplying the columns of $\Xi$ by any value different from zero. Hence, we will refer to the following matrix:
$\Xi^{\prime} \equiv\left[\begin{array}{c|c|c|c|c|c}\mathcal{M}_{2} & \mathcal{P}_{1} & \mathcal{P}_{2} & 0_{3 N} & \ldots & 0_{3 N} \\ \mathcal{M}_{3} & \mathcal{P}_{1} & 0_{3 N} N & \mathcal{P}_{3} & \ldots & 0_{3 N} \\ \ldots & \ldots & \ldots & \ldots & \ldots & \ldots \\ \mathcal{M}_{n_{i}} & \mathcal{P}_{1} & 0_{3 N} N & \ldots & 0_{3 N} & \mathcal{P}_{n_{i}}\end{array}\right]$

where $0_{3 N} N$ denotes the $3 N \times N$ zero matrix and:

$\mathcal{M}_{j} \equiv\left[\begin{array}{c|c|c}T_{j} & S_{j} & \Gamma_{j} \\ 0_{33} & 0_{33} & 0_{33} \\ \ldots & \ldots & \ldots \\ 0_{33} & 0_{33} & 0_{33}\end{array}\right], \quad \mathcal{P}_{j} \equiv\left[\begin{array}{c|c|c|c|c}\boldsymbol{P}_{j}^{1} & 0_{3} & 0_{3} & \ldots & 0_{3} \\ \boldsymbol{P}_{j}^{1} & \boldsymbol{P}_{j}^{2} & 0_{3} & \ldots & 0_{3} \\ \boldsymbol{P}_{j}^{1} & 0_{3} & \boldsymbol{P}_{j}^{3} & \ldots & 0_{3} \\ \ldots & \ldots & \ldots & \ldots & \ldots \\ \boldsymbol{P}_{j}^{1} & 0_{3} & \ldots & 0_{3} & \boldsymbol{P}_{j}^{N}\end{array}\right]$

(note that the last three columns in the matrix $\mathcal{M}_{j}$ disappear in absence of bias). In the following, theorem 1 will be applied by using $\Xi^{\prime}$ instead of $\Xi$. We remark that the difference $\boldsymbol{P}_{j}^{i}-\boldsymbol{P}_{1}^{i}, i=1,2, \ldots, N, j=$ $2, \ldots, n_{i}$, is independent of $i$ (see equation (5), where, by definition, $\left.C_{T_{i n}}^{t_{j}} \boldsymbol{P}_{t_{j}}^{i}\left(t_{j}\right)=\boldsymbol{P}_{j}^{i}\right)$. Hence, we will set $\boldsymbol{\chi}_{j} \equiv \boldsymbol{P}_{j}^{i}-\boldsymbol{P}_{1}^{i}$. This quantity characterizes the motion of the platform.

We will make the following assumption:

Assumption 1 For any $i=1,2, \ldots, N, j=2, \ldots, n_{i}$, $\boldsymbol{P}_{j}^{i} \neq 0_{3}$ (or equivalently, $\chi_{j} \neq-\boldsymbol{P}_{1}^{i}$ ).

This assumption means that during the platform motion, no point-feature can be on the origin of the camera frame. It ensures that no column of $\Xi^{\prime}$ vanishes.

\subsection{Unbiased case}

Let us denote a vector belonging to $\mathcal{N}\left(\Xi^{\prime}\right)$ as follows:

$\boldsymbol{n} \equiv\left[\boldsymbol{\alpha}^{T}, \boldsymbol{\nu}^{T}, n_{1}^{1}, \ldots, n_{1}^{N}, n_{2}^{1}, \ldots, n_{2}^{N}, \ldots, n_{n_{i}}^{1}, \ldots, n_{n_{i}}^{N}\right]^{T}$

where $\boldsymbol{\alpha}$ and $\boldsymbol{\nu}$ are $3 D$ column vectors. $\boldsymbol{n}$ must satisfy:

$\Xi^{\prime} \boldsymbol{n}=0_{3\left(n_{i}-1\right) N}$

where $0_{3\left(n_{i}-1\right) N}$ is the zero $3\left(n_{i}-1\right) N \times 1$ column vector. We can write this system as follows $\left(j=2, \ldots, n_{i}, i=\right.$ $2, \ldots, N)$ :

$$
\begin{array}{r}
-\frac{t_{j}^{2}}{2} \boldsymbol{\alpha}-t_{j} \boldsymbol{\nu}+\left(n_{1}^{1}+n_{j}^{1}\right) \boldsymbol{P}_{1}^{1}+n_{j}^{1} \boldsymbol{\chi}_{j}=0_{3} \\
\left(n_{1}^{1}+n_{j}^{1}\right) \boldsymbol{P}_{1}^{1}+\left(n_{1}^{i}+n_{j}^{i}\right) \boldsymbol{P}_{1}^{i}+\left(n_{j}^{1}+n_{j}^{i}\right) \boldsymbol{\chi}_{j}=0_{3}
\end{array}
$$

We start our analysis by investigating two very special cases: the planar case and the linear case. 


\subsubsection{Planar case}

Let us suppose that all the vectors $\boldsymbol{P}_{j}^{i}, i=1, \ldots, N, j=$ $2, \ldots, n_{i}$, belong to a plane ${ }^{2}$. This means that it exists a frame such that all these vectors have the last component equal to zero. In this new frame the linear system in (13) can be separated in two parts: the former corresponds to the first two lines of (14) and the first two lines of $(15)$ for $j=2, \ldots, n_{i}$; the latter corresponds to the third line of $(14)$ for $j=2, \ldots, n_{i}$, which only involves the third component of $\boldsymbol{\alpha}$ and $\boldsymbol{\nu}$. Let us denote with $\Xi_{1}^{\text {plane }}$ and $\Xi_{2}^{\text {plane }}$ the matrices which characterize these two systems. Their size is $2\left(n_{i}-1\right) N \times\left(N n_{i}+4\right)$ and $\left(n_{i}-1\right) \times 2$, respectively. When $n_{i} \leq 2$, the dimension of $\mathcal{N}\left(\Xi_{1}^{\text {plane }}\right)$ is at least 4 . Hence, from theorem 1 , we obtain that a necessary condition in order to have a finite number of solutions (one or two) is that $n_{i} \geq 3$. The null space of $\Xi_{2}^{\text {plane }}$ has dimension 0 as $n_{i} \geq 3$. Let us consider the case when $n_{i}=3$. The size of $\Xi_{1}^{\text {plane }}$ is $4 N \times(3 N+4)$. Hence, in order to have the dimension of $\mathcal{N}\left(\Xi_{1}^{\text {plane }}\right)$ not larger than 1 it is necessary to have $N \geq 3$. Let us consider the case when $n_{i}=4$. The size of $\Xi_{1}^{\text {plane }}$ is $6 N \times(4 N+4)$. Hence, in order to have the dimension of $\mathcal{N}\left(\Xi_{1}^{\text {plane }}\right)$ not larger than 1 it is necessary to have $N \geq 2$. Finally, when $n_{i} \geq 5$ no necessary condition constrains $N$.

We summarize the results of this subsection with the following property:

Property 1 (Unbiased: Planar Layout) When all the observed point-features and the platform positions are coplanar, a necessary condition to have a finite number of solutions is that $n_{i} \geq 3$. Specifically, if $n_{i}=3$, $N \geq 3$, if $n_{i}=4, N \geq 2$.

\subsubsection{Linear case}

When all the vectors $\boldsymbol{P}_{j}^{i}, i=1, \ldots, N, j=2, \ldots, n_{i}$, belong to a line it exists a frame such that all these vectors have the last two components equal to zero. In this new frame the linear system in (13) can be separated in two parts: the former corresponds to the first line of (14) and the first line of (15) for $j=2, \ldots, n_{i}$; the latter corresponds to the second and third line of (14) for $j=2, \ldots, n_{i}$, which only involve the last two components of $\boldsymbol{\alpha}$ and $\boldsymbol{\nu}$. Let us denote with $\Xi_{1}^{\text {line }}$ and $\Xi_{2}^{\text {line }}$ the matrices which characterize these two systems. Their size is $\left(n_{i}-1\right) N \times\left(N n_{i}+2\right)$ and $2\left(n_{i}-1\right) \times 4$, respectively. The null space of $\Xi_{1}^{\text {line }}$ has dimension at least $N+4$. Hence, the Vi-SfM problem has always

2 This is equivalent to say that the position of any pointfeature and the position of the platform at any time $t_{j}(j=$ $\left.1, \ldots, n_{i}\right)$, are coplanar. infinite solutions. This result is obvious and could be derived in a simpler manner. When the platform motion is on a straight line, any point-feature belonging to this line provides the same bearing data independently of its distance from the platform.

We summarize the results of this subsection with the following property:

Property 2 (Unbiased: Linear Layout) When all the observed point-features and the platform positions are collinear, the Vi-SfM problem has always infinite solutions. Additionally, when the platform motion is on a straight line, it is not possible to determine the distance of all the point-features belonging to this line even if there are other point-features outside the line.

Let us consider now the general $3 D$ case. We have the following property:

Property 3 When $n_{i} \leq 2$ the dimension of $\mathcal{N}\left(\Xi^{\prime}\right)$ is at least 3. When $n_{i}=3$ the dimension of $\mathcal{N}\left(\Xi^{\prime}\right)$ is at least 1. Finally, when $n_{i} \geq 4$ and the platform moves with constant acceleration the dimension of $\mathcal{N}\left(\Xi^{\prime}\right)$ is at least 1 .

Proof : In order to prove all these three statements we need to focus our attention on the following subsystem:

$-\frac{t_{j}^{2}}{2} \boldsymbol{\alpha}-t_{j} \boldsymbol{\nu}=-\chi_{j}, \quad j=2, \ldots, n_{i}$

Let us denote the matrix characterizing this linear system with $\Xi^{\prime \prime}$. It is immediate to realize that the dimension of $\mathcal{N}\left(\Xi^{\prime}\right)$ is never smaller than the dimension of $\mathcal{N}\left(\Xi^{\prime \prime}\right)$. Indeed, if the vector $\left[\boldsymbol{n}_{\boldsymbol{\alpha}}{ }^{T}, \boldsymbol{n}_{\boldsymbol{\nu}}^{T}\right]^{T} \in \mathcal{N}\left(\Xi^{\prime \prime}\right)$ then the vector in (12) with $\boldsymbol{\alpha}=\boldsymbol{n}_{\boldsymbol{\alpha}}, \boldsymbol{\nu}=\boldsymbol{n}_{\boldsymbol{\nu}}$, and $n_{j}^{i}=0, \forall i, j$, belongs to $\mathcal{N}\left(\Xi^{\prime}\right)$. The first statement is a consequence of the fact that the dimension of $\mathcal{N}\left(\Xi^{\prime \prime}\right)$ is at least 3 when $n_{i} \leq 2$.

Let us consider the case of $n_{i}=3$. The linear system in (16) can always be solved, independently of the platform motion (i.e., for any set of vectors $\chi_{j}$ ). In particular, the equations in (16) for $j=2,3$ form a linear square system, which has a unique solution, $\left(\boldsymbol{\alpha}_{0}, \boldsymbol{\nu}_{0}\right)$. From (14-16) we obtain that the vector in (12) with $\boldsymbol{\alpha}=\boldsymbol{\alpha}_{0}, \boldsymbol{\nu}=\boldsymbol{\nu}_{0}, n_{1}^{1}=\bar{n}_{1}^{1} \equiv-1, n_{j}^{1}=\bar{n}_{j}^{1} \equiv 1$, $n_{1}^{i}=\bar{n}_{1}^{i} \equiv 1, n_{j}^{i}=\bar{n}_{j}^{i} \equiv-1(j=2,3 ; i=2, \ldots, N)$ belongs to $\mathcal{N}\left(\Xi^{\prime}\right)$ when $n_{i}=3$. We will denote this vector with $\boldsymbol{n}_{0}$ :

$\boldsymbol{n}_{0} \equiv\left[\boldsymbol{\alpha}_{0}, \boldsymbol{\nu}_{0}, \bar{n}_{1}^{1}, . . \bar{n}_{1}^{i} . ., . . \bar{n}_{j}^{1} . ., . . \bar{n}_{j}^{i} . .\right]^{T}$

Hence, when $n_{i}=3$ the vector $\boldsymbol{n}_{0} \in \mathcal{N}\left(\Xi^{\prime}\right)$ for any motion and the dimension of $\mathcal{N}\left(\Xi^{\prime}\right)$ is at least 1 . 
Finally, the system in (16) can be solved for any $n_{i} \geq 4$ if and only if $\boldsymbol{\chi}_{j}=\boldsymbol{\nu}_{0} t_{j}+\boldsymbol{\alpha}_{0} \frac{t_{j}^{2}}{2}$. This situation corresponds to a platform motion with constant acceleration $\boldsymbol{\alpha}_{0}$ and initial speed $\boldsymbol{\nu}_{0}$. Hence, also in this case $\boldsymbol{n}_{0} \in \mathcal{N}\left(\Xi^{\prime}\right)$

In order to apply theorem 1 , we need to understand if $\boldsymbol{n}_{0}$ is the only generator of $\mathcal{N}\left(\Xi^{\prime}\right)$, i.e., if $\mathcal{N}\left(\Xi^{\prime}\right)$ has dimension equal or larger than 1 .

\section{$4.1 .3 n_{i} \leq 2$}

From property 3 we know that the dimension of $\mathcal{N}\left(\Xi^{\prime}\right)$ is at least 3 and, consequently, the Vi-SfM problem has always infinite solutions.

\section{$4.1 .4 n_{i}=3$}

From property 3 we know that the dimension of $\mathcal{N}\left(\Xi^{\prime}\right)$ is at least 1 , independently of the number of pointfeatures. When $N=1, \Xi^{\prime}$ is a $6 \times 9$ matrix. Hence, the dimension of $\mathcal{N}\left(\Xi^{\prime}\right)$ is at least 3 . Let us consider the case when $N=2$. In this case $\Xi^{\prime}$ is a $12 \times 12$ matrix. We have the following property:

Property 4 (Minimal case: $n_{\boldsymbol{i}}=\mathbf{3}, \boldsymbol{N}=\mathbf{2}$ ) The $d i$ mension of $\mathcal{N}\left(\Xi^{\prime}\right)$ is 1 if and only if the following two conditions are met:

(i) for a given $j$ (e.g., for $j=2$ ), the three vectors $\boldsymbol{P}_{1}^{1}, \boldsymbol{P}_{1}^{2}$ and $\boldsymbol{\chi}_{j}$ span the entire $3 D$-space;

(ii) for the other value of $j$ (e.g., for $j=3$ ) $\boldsymbol{P}_{j}^{i}$ is not proportional to $\boldsymbol{P}_{j}^{k}, \forall i, k=1,2, \ldots, N$.

Otherwise, the dimension of $\mathcal{N}\left(\Xi^{\prime}\right)$ is larger than 1.

Proof : If (i) is not true, all the vectors $\boldsymbol{P}_{j}^{i}, i=1,2, j=$ 2,3 , belong to a plane. Since $N=2$, the dimension of $\mathcal{N}\left(\Xi^{\prime}\right)$ is larger than 1 (see property 1). Let us suppose now that the condition (i) is met for $j=2$. From (15) with $j=2$ we obtain: $n_{1}^{1}=n_{2}^{2}=-n_{2}^{1}=-n_{1}^{2}$. From (14) with $j=2$ we obtain the same equation in (16) with $n_{2}^{1} \chi_{2}$ instead of $\chi_{2}$. From (15) with $j=3$ we obtain: $\left(-n_{2}^{1}+n_{3}^{1}\right) \boldsymbol{P}_{3}^{1}=\left(n_{2}^{1}+n_{3}^{2}\right) \boldsymbol{P}_{3}^{2}$. If the condition (ii) is met, we have: $n_{3}^{1}=-n_{3}^{2}=n_{2}^{1}$ and from (14) with $j=3$ we obtain the same equation in (16) with $n_{2}^{1} \chi_{3}$ instead of $\chi_{3}$. In other words, when the condition (ii) is met we have the same equations as in (16) for $j=2,3$, with $n_{2}^{1} \chi_{j}$ instead of $\chi_{j}$. As previously mentioned, this system has a unique solution and $\mathcal{N}\left(\Xi^{\prime}\right)$ is generated by $\boldsymbol{n}_{0}$. If the condition (ii) is not met, equation $\left(-n_{2}^{1}+n_{3}^{1}\right) \boldsymbol{P}_{3}^{1}=\left(n_{2}^{1}+n_{3}^{2}\right) \boldsymbol{P}_{3}^{2}$ has further solutions and consequently $\boldsymbol{n}_{0}$ is not the only generator of $\mathcal{N}\left(\Xi^{\prime}\right)$

From now on, we will say that a condition is satisfied in general when the probability that it is not satisfied is zero. We remark that both conditions (i) and (ii) are met in general.

Also for $N>2$ there are still conditions, which occur with zero probability, under which the dimension of $\mathcal{N}\left(\Xi^{\prime}\right)$ is larger than 1 . We summarize the results of this subsection with the following property:

Property 5 (Unbiased with $\boldsymbol{n}_{\boldsymbol{i}}=\mathbf{3}, \boldsymbol{N} \geq \mathbf{2}$ ) When $n_{i}=3$ and $N \geq 2$ the Vi-SfM problem has in general two distinct solutions. In some special cases it has infinite solutions.

\section{$4.1 .5 n_{i} \geq 4$}

When $n_{i} \geq 4$ the number of equations is larger than the number of unknowns, except when $n_{i}=4$ and $N=1$. In this case the matrix $\Xi^{\prime}$ is $9 \times 10$ and the dimension of its null space is at least 1 . We have the following property:

Property 6 (Minimal case: $n_{i}=4, N=1$ ) The $d i$ mension of $\mathcal{N}\left(\Xi^{\prime}\right)$ is 1 if and only if the four vectors $\boldsymbol{P}_{1}^{1}, \chi_{2}, \chi_{3}$ and $\chi_{4}$ span the entire $3 D$-space.

Proof : If the vectors $\boldsymbol{P}_{1}^{1}$ and $\boldsymbol{\chi}_{j}, j=2,3,4$, are coplanar, since $N=1$, the dimension of $\mathcal{N}\left(\Xi^{\prime}\right)$ is larger than 1 (see property 1). Let us suppose now that the vectors $\boldsymbol{P}_{1}^{1}$ and $\boldsymbol{\chi}_{j}, j=2,3,4$ span the entire $3 D$-space. From the first 6 equations in (13) (i.e., the equation (14) for $j=2,3)$ we obtain $\boldsymbol{\alpha}$ and $\boldsymbol{\nu}$ as linear functions of $\boldsymbol{P}_{1}^{1}, \chi_{2}$ and $\boldsymbol{\chi}_{3}$. By substituting the expressions of $\boldsymbol{\alpha}$ and $\boldsymbol{\nu}$ in the last three equations (i.e., in (14) with $j=4$ ) we obtain the following equation: $a_{1} \boldsymbol{P}_{1}^{1}+a_{2} \boldsymbol{\chi}_{2}+a_{3} \boldsymbol{\chi}_{3}+a_{4} \boldsymbol{\chi}_{4}=0_{3}$, where $a_{1}, a_{2}, a_{3}, a_{4}$ are linear expressions of $n_{1}^{1}, n_{2}^{1}, n_{3}^{1}, n_{4}^{1}$. Since the four vectors span the entire $3 D-$ space, the null space of the $3 \times 4$ matrix $\left[\boldsymbol{P}_{1}^{1}, \chi_{2}, \chi_{3}, \chi_{4}\right]$ has dimension 1 . Let us denote with $\left[a_{1}^{*}, a_{2}^{*}, a_{3}^{*}, a_{4}^{*}\right]^{T}$ a generator of this null space. We consider the linear system $a_{k}\left(n_{1}^{1}, n_{2}^{1}, n_{3}^{1}, n_{4}^{1}\right)=a_{k}^{*}$, $k=1,2,3,4$. We analytically compute the determinant of the $4 \times 4$ matrix, which characterizes this linear system. We obtain: $\frac{-\left(t_{4}-t_{3}\right)^{2}\left(t_{4}-t_{2}\right)^{2} t_{4}^{2}}{\left(t_{2}-t_{3}\right)^{2} t_{3}^{2} t_{2}^{2}}$. This determinant is always different from 0 (note that $0<t_{2}<t_{3}<t_{4}$ ). Hence, the previous linear system provides a unique solution and the dimension of $\mathcal{N}\left(\Xi^{\prime}\right)$ is 1

We do not derive necessary and sufficient conditions for any value of $n_{i}$ and $N$. The following property holds:

Property 7 (Unbiased with $\boldsymbol{n}_{\boldsymbol{i}} \geq 4$ ) When $n_{i}=4$ and $N=1$ the Vi-SfM problem has in general two distinct solutions. If $n_{i}=4, N \geq 2$ or if $n_{i} \geq 5, \forall N$ it has in general a unique solution. 
Proof : Since the four vectors $\boldsymbol{P}_{1}^{1}, \chi_{2}, \chi_{3}$ and $\chi_{4}$ span in general the entire $3 D$-space, property 6 proves the first statement.

To prove the second part we start by considering the case $n_{i} \geq 4$ and $N \geq 2$. In general, the three vectors $\boldsymbol{P}_{1}^{1}$, $\boldsymbol{P}_{1}^{i}$ and $\chi_{j}$ are independent for each $i=2, \ldots, N$ and for each $j=2, \ldots, n_{i}$. Hence, from equation (15) we obtain: $n_{1}^{1}+n_{j}^{1}=n_{1}^{i}+n_{j}^{i}=n_{j}^{1}+n_{j}^{i}=0, \forall i \geq 2, \forall j \geq 2$. If $n_{1}^{1} \neq 0$, let us set, without loss of generality, $n_{1}^{1}=1$. Equation (14) becomes: $-\frac{t_{j}^{2}}{2} \boldsymbol{\alpha}-t_{j} \boldsymbol{\nu}=\chi_{j}$. For $n_{i} \geq 4$ this equation does not hold in general since it only holds for a motion with constant acceleration (this special case will be dealt in more detail in 4.1.6). Hence, $n_{1}^{1}=0$ and, consequently, $n_{j}^{i}=0 \forall i, \forall j$. From (14) we also have $\boldsymbol{\alpha}=\boldsymbol{\nu}=0_{3}$. Therefore, the dimension of $\mathcal{N}\left(\Xi^{\prime}\right)$ is 0 .

Let us now consider the case $n_{i} \geq 5$ and $N=1$. From property 6 we know that, in general, it exists one independent vector $\hat{\boldsymbol{n}}_{4} \equiv\left[\hat{\boldsymbol{\alpha}}^{T}, \hat{\boldsymbol{\nu}}^{T}, \hat{n}_{1}^{1}, \hat{n}_{2}^{1}, \hat{n}_{3}^{1}, \hat{n}_{4}^{1}\right]^{T}$ satisfying equation (14) for $j=2,3,4$. Hence, any solution of (13) must have the first ten components coincident with the ones of $\hat{\boldsymbol{n}}_{4}$ or all the first ten components equal to zero. Let us consider a given $j \geq 5$. In the former case (i.e., first ten components equal to $\hat{\boldsymbol{n}}_{4}$ ), equation (14) reads as follows: $-\frac{t_{j}^{2}}{2} \hat{\boldsymbol{\alpha}}-t_{j} \hat{\boldsymbol{\nu}}+\left(\hat{n}_{1}^{1}+n_{j}^{1}\right) \boldsymbol{P}_{1}^{1}+n_{j}^{1} \boldsymbol{\chi}_{j}=$ $0_{3}$. This equation does not hold in general. Indeed, if $n_{j}^{1}=0,-\frac{t_{j}^{2}}{2} \hat{\boldsymbol{\alpha}}-t_{j} \hat{\boldsymbol{\nu}}+\hat{n}_{1}^{1} \boldsymbol{P}_{1}^{1}=0_{3}$ (which is not true in general) and, if $n_{j}^{1} \neq 0$, the vector $\boldsymbol{P}_{1}^{1}+\boldsymbol{\chi}_{j}$ must be parallel to the vector $-\frac{t_{j}^{2}}{2} \hat{\boldsymbol{\alpha}}-t_{j} \hat{\boldsymbol{\nu}}+\hat{n}_{1}^{1} \boldsymbol{P}_{1}^{1}$ (which is not the case in general). In the latter case (i.e., all the first ten components equal to zero), equation (14) reads as follows: $n_{j}^{1}\left(\boldsymbol{P}_{1}^{1}+\boldsymbol{\chi}_{j}\right)=0_{3}$. Because of assumption 1 this holds if and only if $n_{j}^{1}=0$

\subsubsection{Constant acceleration}

Let us consider the case when the platorm moves with constant acceleration, i.e. when $\chi_{j}=\boldsymbol{\nu}_{0} t_{j}+\boldsymbol{\alpha}_{0} \frac{t_{j}^{2}}{2}, j=$ $2, \ldots, n_{i}$, where $\boldsymbol{\nu}_{0}$ and $\boldsymbol{\alpha}_{0}$ are two $3 D$-vectors. We already know from property 3 that the dimension of $\mathcal{N}\left(\Xi^{\prime}\right)$ is at least 1 . Specifically, the vector $\boldsymbol{n}_{0}$ in (17) belongs to the null space of $\Xi^{\prime}$. In order to use theorem 1 , we need to understand when $\mathcal{N}\left(\Xi^{\prime}\right)$ has dimension equal or larger than 1 . The following property provides a sufficient condition which ensures the Vi-SfM resolvability.

\section{Property 8 (Unbiased with constant acceleration)} Let us suppose that the platform moves with constant acceleration, i.e., $\boldsymbol{\chi}_{j}=\boldsymbol{\nu}_{0} t_{j}+\boldsymbol{\alpha}_{0} \frac{t_{j}^{2}}{2}, j=2, \ldots, n_{i}$. When for a given point-feature $k$ the vectors $\boldsymbol{\nu}_{0}, \boldsymbol{\alpha}_{0}$ and $\boldsymbol{P}_{1}^{k}$ span the entire $3 D$-space the dimension of $\mathcal{N}\left(\Xi^{\prime}\right)$ is 1 .
Proof : Without loss of generality, let us set $k=1$. From the first 6 equations in (13) (i.e., the equation (14) for $j=2,3)$ we obtain $\boldsymbol{\alpha}$ and $\boldsymbol{\nu}$ as linear functions of $\boldsymbol{P}_{1}^{1}, \boldsymbol{\alpha}_{0}$ and $\boldsymbol{\nu}_{0}$. By substituting the expressions of $\boldsymbol{\alpha}$ and $\boldsymbol{\nu}$ in (14) with $j=4$ we obtain the following equation: $a_{1} \boldsymbol{P}_{1}^{1}+a_{2} \boldsymbol{\alpha}_{0}+a_{3} \boldsymbol{\nu}_{0}=0_{3}$, where $a_{1}, a_{2}, a_{3}$ are linear expressions of $n_{1}^{1}, n_{2}^{1}, n_{3}^{1}, n_{4}^{1}$. Since the three vectors span the entire $3 D$-space, we must have $a_{k}\left(n_{1}^{1}, n_{2}^{1}, n_{3}^{1}, n_{4}^{1}\right)=0, k=1,2,3$. This linear system is characterized by a $3 \times 4$ matrix. Hence, it has at least one non trivial solution. By a direct computation, it is possible to see that the dimension of the null space of this matrix is 1 . The non trivial solution is $n_{1}^{1}=-1, n_{2}^{1}=n_{3}^{1}=n_{4}^{1}=1$. Now, let us consider the equation $(15)$. We obtain $(i \neq 1)$ :

$\left(n_{1}^{i}+n_{j}^{i}\right) \boldsymbol{P}_{1}^{i}+\left(1+n_{j}^{i}\right)\left(\boldsymbol{\nu}_{0} t_{j}+\boldsymbol{\alpha}_{0} \frac{t_{j}^{2}}{2}\right)=0_{3}$

On the other hand, a further consequence of the fact that $\boldsymbol{\nu}_{0}, \boldsymbol{\alpha}_{0}$ and $\boldsymbol{P}_{1}^{1}$ span the entire $3 D$-space, is that the two vectors $\boldsymbol{\nu}_{0}$ and $\boldsymbol{\alpha}_{0}$ cannot be collinear. Hence, it exists a value of $j=j^{*}$, such that $\boldsymbol{P}_{1}^{i}$ is not proportional to $\boldsymbol{\nu}_{0} t_{j^{*}}+\boldsymbol{\alpha}_{0} \frac{t_{j^{*}}^{2}}{2}$. From (18) we immediately obtain $n_{j^{*}}^{i}=-1$ and $n_{1}^{i}=1$. For the other $j \neq j^{*}$ we obtain: $\left(1+n_{j}^{i}\right)\left(\boldsymbol{P}_{1}^{i}+\boldsymbol{\nu}_{0} t_{j}+\boldsymbol{\alpha}_{0} \frac{t_{j}^{2}}{2}\right)=0_{3}$. If $n_{j}^{i} \neq-1$ $\boldsymbol{P}_{1}^{i}=-\boldsymbol{\nu}_{0} t_{j}-\boldsymbol{\alpha}_{0} \frac{t_{j}^{2}}{2}=-\boldsymbol{\chi}_{j}$, which is not possible because of the assumption 1

This property ensures that, when the platform moves with constant acceleration, the Vi-SfM problem has in general two solutions.

A special case of constant acceleration occurs when the vector $\boldsymbol{\alpha}_{0}$ vanishes, i.e., when the platform moves with constant speed. Since $\left|\Pi \boldsymbol{n}_{0}\right|=\left|\boldsymbol{\alpha}_{0}\right|=0$, according to theorem 1, the Vi-SfM has infinite solutions. However, as it has been proven at the end of the proof of that theorem, in this case the local gravity $\boldsymbol{G}$ can be uniquely determined. Hence, the orientation of the platform with respect to the horizontal plane can be uniquely determined. We proved the following property:

Property 9 (Unbiased with constant speed) Let us suppose that the platform moves with constant speed. The Vi-SfM has infinite solutions. Additionally, the orientation of the platform with respect to the horizontal plane can be uniquely determined.

\subsection{Biased case}

We investigate now the resolvability of the Vi-SfM problem when the accelerometers are affected by a bias. Obviously, all the necessary conditions derived in 4.1 are 
still necessary in this harder case. On the other hand, there are cases where conditions which ensure resolvability in the unbiased case, are no longer sufficient in this case. By proceeding as in the unbiased case (see (12)), we will denote a vector belonging to $\mathcal{N}\left(\Xi^{\prime}\right)$ as follows:

$\boldsymbol{n} \equiv\left[\boldsymbol{\alpha}^{T}, \boldsymbol{\nu}^{T}, \boldsymbol{b}^{T}, n_{1}^{1}, \ldots, n_{1}^{N}, n_{2}^{1}, \ldots, n_{2}^{N}, \ldots, n_{n_{i}}^{1}, \ldots, n_{n_{i}}^{N}\right]^{T}$

where $\boldsymbol{b}$ is a $3 D$-vector (as $\boldsymbol{\alpha}$ and $\boldsymbol{\nu}$ ). $\boldsymbol{n}$ must satisfy (13) where now $\Xi^{\prime}$ also includes the third set of columns. We can write this system as in (14-15). In this case, the first equation must be replaced by:

$-\frac{t_{j}^{2}}{2} \boldsymbol{\alpha}-t_{j} \boldsymbol{\nu}+\Gamma_{j} \boldsymbol{b}+\left(n_{1}^{1}+n_{j}^{1}\right) \boldsymbol{P}_{1}^{1}+n_{j}^{1} \chi_{j}=0_{3}$

Regarding the planar and linear cases, properties 1 and 2 still hold since they only provide necessary conditions. However, regarding the planar case, more restrictive conditions can be derived, which even hold in the $3 D-$ case $^{3}$.

In the biased case, property 3 is replaced by the following property:

Property 10 When $n_{i} \leq 3$ the dimension of $\mathcal{N}\left(\Xi^{\prime}\right)$ is at least 3 . When $n_{i}=4$ the dimension of $\mathcal{N}\left(\Xi^{\prime}\right)$ is at least 1. Finally, when $n_{i} \geq 5$ and the platform moves with the special motion characterized by $\chi_{j}=$ $-\frac{t_{j}^{2}}{2} \boldsymbol{\alpha}_{0}-t_{j} \boldsymbol{\nu}_{0}+\Gamma_{j} \boldsymbol{b}_{0}$, for three vectors $\boldsymbol{\alpha}_{0}, \boldsymbol{\nu}_{0}$ and $\boldsymbol{b}_{0}$ and $j \geq 5$, the dimension of $\mathcal{N}\left(\Xi^{\prime}\right)$ is at least 1 .

Proof : In order to prove these three statements we need to focus our attention on the following subsystem:

$-\frac{t_{j}^{2}}{2} \boldsymbol{\alpha}-t_{j} \boldsymbol{\nu}+\Gamma_{j} \boldsymbol{b}=-\chi_{j}, \quad j=2, \ldots, n_{i}$

Let us denote the matrix characterizing this linear system with $\Xi_{b}^{\prime \prime}$. It is immediate to realize that the dimension of $\mathcal{N}\left(\Xi^{\prime}\right)$ is never smaller than the dimension of $\mathcal{N}\left(\Xi_{b}^{\prime \prime}\right)$. Indeed, if the vector $\left[\boldsymbol{n}_{\boldsymbol{\alpha}}{ }^{T}, \boldsymbol{n}_{\boldsymbol{\nu}}{ }^{T}, \boldsymbol{n}_{\boldsymbol{b}}{ }^{T}\right]^{T} \in$ $\mathcal{N}\left(\Xi_{b}^{\prime \prime}\right)$ then the vector in (19) with $\boldsymbol{\alpha}=\boldsymbol{n}_{\boldsymbol{\alpha}}, \boldsymbol{\nu}=\boldsymbol{n}_{\boldsymbol{\nu}}$, $\boldsymbol{b}=\boldsymbol{n}_{\boldsymbol{b}}$ and $n_{j}^{i}=0, \forall i, j$, belongs to $\mathcal{N}\left(\Xi^{\prime}\right)$. The first statement is a consequence of the fact that the dimension of $\mathcal{N}\left(\Xi_{b}^{\prime \prime}\right)$ is at least 3 when $n_{i} \leq 3$ (being the number of equations in (21) not more than 6 and the number of unknowns is 9 ).

\footnotetext{
3 Note that it is not possible to proceed as in the unbiased case since it is not possible to separate the linear system in (13) in two parts because of the bias.
}

Let us consider the case of $n_{i}=4 . \Xi_{b}^{\prime \prime}$ is a square matrix. We distinguish two cases: the case when the determinant of $\Xi_{b}^{\prime \prime}$ vanishes and the case when is nonzero. In the first case the dimension of $\mathcal{N}\left(\Xi_{b}^{\prime \prime}\right)$ is at least 1 and, as shown in the first part of this proof, also the dimension of $\mathcal{N}\left(\Xi^{\prime}\right)$ is at least 1 . In the second case, the linear system in (21) can be uniquely solved (for any set of vectors $\left.\boldsymbol{\chi}_{j}\right)$. Let us denote this solution with $\left(\boldsymbol{\alpha}_{0}, \boldsymbol{\nu}_{0}\right.$, $\left.\boldsymbol{b}_{0}\right)$. From (15) and (20) we obtain that the vector in (19) with $\boldsymbol{\alpha}=\boldsymbol{\alpha}_{0}, \boldsymbol{\nu}=\boldsymbol{\nu}_{0}, \boldsymbol{b}=\boldsymbol{b}_{0}, n_{1}^{1}=n_{j}^{i}=-1$, $n_{j}^{1}=n_{1}^{i}=1,(j=2,3,4, i=2, \ldots, N)$ belongs to $\mathcal{N}\left(\Xi^{\prime}\right)$. Hence, also in this case the dimension of $\mathcal{N}\left(\Xi^{\prime}\right)$ is at least 1 .

Finally, the system in (21) can be solved for $n_{i} \geq 5$ if and only if the platform motion satisfies the equation $\boldsymbol{\chi}_{j}=-\frac{t_{j}^{2}}{2} \boldsymbol{\alpha}_{0}-t_{j} \boldsymbol{\nu}_{0}+\Gamma_{j} \boldsymbol{b}_{0}$. In this case, the vector $\boldsymbol{n}_{0}$ in (17) becomes:

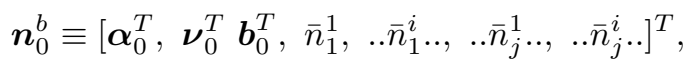

and it is immediate to verify that $\boldsymbol{n}_{0}^{b} \in \mathcal{N}\left(\Xi^{\prime}\right)$

Note that, when $\boldsymbol{b}_{0}=0_{3}$, the special motion considered in this property is the motion with constant acceleration defined in the unbiased case.

We remark that, in the unbiased case, the platform rotations do not affect the problem resolvability. Indeed, in the matrix $\Xi^{\prime}$, only the third set of columns are affected by the platform rotations. In the biased case it is easy to prove the following property:

Property 11 (Biased: impact of rotations, part 1) When the platform does not rotate the dimension of $\mathcal{N}\left(\Xi^{\prime}\right)$ is at least 3 . When the platform rotates always around the same axis the dimension of $\mathcal{N}\left(\Xi^{\prime}\right)$ is at least 1.

Proof: When the platform does not rotate $\Gamma_{j}=\frac{t_{j}^{2}}{2} I_{3}$ (see the definition of $\Gamma_{j}$ in (2)). Hence, the third set of columns coincides with the first set up to a sign. This means that the dimension of $\mathcal{N}\left(\Xi^{\prime}\right)$ is at least 3. Let us consider the case when the rotations only occur around the same axis. We can assume without loss of generality that it is the $z$-axis (indeed, we can change the camera frame in such a way that its new $z$-axis is aligned with the axis of rotation). From the definition of $\Gamma$ in (2) we remark that, in this case, the third column of $\Gamma_{j}$ coincides with the third column of $T_{j}$ in (8) up to a sign. Hence, the vector in (19) with all the entries zero with the exception of the third and the ninth entry equal one each other, belongs to $\mathcal{N}\left(\Xi^{\prime}\right)$

It also holds the following stronger property: 
Property 12 (Biased: impact of rotations, part 2) When the platform rotates always around the same axis the dimension of $\mathcal{N}\left(\Xi_{b}^{\prime \prime}\right)$ is in general 1 (provided that $\left.n_{i} \geq 4\right)$. When the platform rotates around at least two independent axes the dimension of $\mathcal{N}\left(\Xi_{b}^{\prime \prime}\right)$ is in general 0 (provided that $n_{i} \geq 4$ ).

Proof : The proof of this property is much more troublesome than the proof of property 11. It becomes easier by assuming that the observations are provided continuously in time (i.e., if the discrete index $j$ in (21) is replaced by the continuous index $t$ ). In the following, for the sake of clarity, we prove the two statements under this ideal assumption (which we will call the continuous assumption). Finally, we prove the first statement in the discrete case.

Under the continuous assumption, by differentiating three times equation (21) with respect to time we obtain: $\frac{d^{3} \Gamma(t)}{d t^{3}} \boldsymbol{b}=-\frac{d^{3} \boldsymbol{\chi}(\mathrm{t})}{d t^{3}}$. From the definition of $\Gamma(t)$ in (2) we obtain (we remind the reader that we set $\left.T_{\text {in }}=0\right)$ :

$\frac{d C_{0}^{t}}{d t} \boldsymbol{b}=-\frac{d^{3} \chi(\mathrm{t})}{d t^{3}}$

Since $C_{0}^{t}$ is the rotation matrix generated by the angular speed $\boldsymbol{\Omega}(t)$, we also have: $\frac{d C_{0}^{t}}{d t}=[\boldsymbol{\Omega}(t)]_{\times} C_{0}^{t}$, where $[\boldsymbol{\Omega}(t)]_{\times} \equiv\left[\begin{array}{ccc}0 & -\Omega_{z} & \Omega_{y} \\ \Omega_{z} & 0 & -\Omega_{x} \\ -\Omega_{y} & \Omega_{x} & 0\end{array}\right]$. By using this equation in (23) and by denoting $\boldsymbol{b}^{\prime} \equiv C_{0}^{t} \boldsymbol{b}$ we obtain:

$[\boldsymbol{\Omega}(t)]_{\times} \boldsymbol{b}^{\prime}=-\frac{d^{3} \chi(\mathrm{t})}{d t^{3}}$

For a non-zero $\boldsymbol{\Omega}(t)$, the previous system has rank 2 . Hence, it allows us to determine two components of $\boldsymbol{b}$ in terms of the third one. Additionally, by considering the system in (21) at two distinct times, it is possible to uniquely obtain the vectors $\boldsymbol{\alpha}$ and $\boldsymbol{\nu}$ in terms of $\boldsymbol{b}$. Hence, the dimension of $\mathcal{N}\left(\Xi_{b}^{\prime \prime}\right)$ is at most 1 . On the other hand, by proceeding as in the proof of property 11 it is possible to show that, when the platform rotates always around the same axis, the dimension of $\mathcal{N}\left(\Xi_{b}^{\prime \prime}\right)$ is at least 1 . Therefore, the dimension of $\mathcal{N}\left(\Xi_{b}^{\prime \prime}\right)$ is 1 .

When the platform rotates around at least two independent axes, by taking (24) at two distinct times (where the two corresponding angular velocities are not proportional), we can determine all the three components of $\boldsymbol{b}$. By considering the system in (21) at two distinct times, it is possible to uniquely obtain the vectors $\boldsymbol{\alpha}$ and $\boldsymbol{\nu}$ in terms of $\boldsymbol{b}$, which is now determined. Hence, the dimension of $\mathcal{N}\left(\Xi_{b}^{\prime \prime}\right)$ is 0 .
We conclude this proof by considering the realistic discrete case and by proving only the first statement. By proceeding as in the proof of property 11 it is possible to show that, when the platform rotates always around the same axis, the dimension of $\mathcal{N}\left(\Xi_{b}^{\prime \prime}\right)$ is at least 1 . Hence, it sufficies to show that when $n_{i}=4$ the dimension of $\mathcal{N}\left(\Xi_{b}^{\prime \prime}\right)$ is in general 1 . The matrix $\Xi_{b}^{\prime \prime}$ is in this case:

$\Xi_{b}^{\prime \prime}=\left[\begin{array}{ccc}T_{2} & S_{2} & \Gamma_{2} \\ T_{3} & S_{3} & \Gamma_{3} \\ T_{4} & S_{4} & \Gamma_{4}\end{array}\right]$

By doing a Gauss elimination it is immediate to verify that the dimension of the null space of this matrix is equal to the dimension of the null space of the following $3 \times 3$ matrix:

$\Gamma^{\prime} \equiv w_{23} \Gamma_{4}+w_{24} \Gamma_{3}+w_{34} \Gamma_{2}$

where $w_{23}=t_{2} t_{3}^{2}-t_{2}^{2} t_{3}, w_{24}=t_{4} t_{2}^{2}-t_{4}^{2} t_{2}$ and $w_{34}=$ $t_{3} t_{4}^{2}-t_{3}^{2} t_{4}$. On the other hand, by setting, without loss of generality, the $z$-axis as the rotation axis, the matrix $\Gamma_{j}$ has the structure: $\left[\begin{array}{ccc}c_{j} & s_{j} & 0 \\ -s_{j} & c_{j} & 0 \\ 0 & 0 & \frac{t_{j}^{2}}{2}\end{array}\right]$ where $c_{j} \equiv \int_{0}^{t_{j}}\left(t_{j}-\right.$ $\tau) \cos \theta(\tau) d \tau, s_{j} \equiv \int_{0}^{t_{j}}\left(t_{j}-\tau\right) \sin \theta(\tau) d \tau$ and $\theta(\tau)$ is the rotation accomplished by the platform up to time $\tau$. By using this expression in (26) we obtain that the third line of $\Gamma^{\prime}$ vanishes. In order to show that the dimension of $\mathcal{N}\left(\Gamma^{\prime}\right)$ is in general 1 it suffices to prove that the following expression is in general different from zero: $w_{23} c_{4}+w_{24} c_{3}+w_{34} c_{2}$. We show that this expression is in general different from zero in the following infinite dimensional space of continuous function $\mathcal{V} \equiv\left\{f \in \mathcal{C}^{1}\right.$ : $\left.\left[T_{i n}=0, T_{f i n}\right] \rightarrow[-1,1]\right\}$, i.e., that in this space the following equation holds in general ${ }^{4}$ :

$w_{23} \int_{0}^{t_{4}}\left(t_{4}-\tau\right) f(\tau) d \tau+w_{24} \int_{0}^{t_{3}}\left(t_{3}-\tau\right) f(\tau) d \tau+$

$w_{34} \int_{0}^{t_{2}}\left(t_{2}-\tau\right) f(\tau) d \tau \neq 0$

A probe for this is the one-dimensional space of all the constant function in $[-1,1]$. This proves that the set $\mathcal{T} \subset \mathcal{V}$ where $(27)$ holds is prevalent (see [12] for the definition of probe and prevalence )

4 Note that we are not considering the space of the functions $\theta(\tau)$ but the space of the functions $\cos \theta(\tau)$. 


\subsection{1 $n_{i} \leq 3$}

From property 10 we know that the dimension of $\mathcal{N}\left(\Xi^{\prime}\right)$ is at least 3 . Hence, the following property holds:

Property 13 (Biased case, $\boldsymbol{n}_{\boldsymbol{i}} \leq \mathbf{3}$ ) The Vi-SfM problem has always infinite solutions in the biased case when $n_{i} \leq 3$.

\subsection{2 $n_{i}=4$}

From property 10 we know that the dimension of $\mathcal{N}\left(\Xi^{\prime}\right)$ is at least 1 . In order to apply theorem 1 we need to know when it is exactly 1 , in which case the ViSfM problem has two distinct solutions. We have the following property:

Property 14 (Biased case, $\boldsymbol{n}_{\boldsymbol{i}}=4$ ) In the biased case when $n_{i}=4$ the Vi-SfM problem has always infinite solutions if $N=1$ and in general two distinct solutions if $N \geq 2$ and the platform rotates around at least one axis.

Proof : Proving the first statement is trivial since for $N=1$, the number of unknowns in (9) is 13 and the number of equations is 9 . When $N \geq 2$ the number of unknowns is smaller than the number of equations. We will prove that, when $N \geq 2$, the dimension of $\mathcal{N}\left(\Xi^{\prime}\right)$ is in general 1 both if the platform rotates around a single axis and if it rotates around two or more axes.

In general, the three vectors $\boldsymbol{P}_{1}^{1}, \boldsymbol{P}_{1}^{i}$ and $\boldsymbol{\chi}_{j}$ are independent for each $i=2, \ldots, N$ and for each $j=$ $2, \ldots, n_{i}$. Hence, from equation (15) we obtain: $n_{1}^{1}+$ $n_{j}^{1}=n_{1}^{i}+n_{j}^{i}=n_{j}^{1}+n_{j}^{i}=0, \forall i \geq 2, \forall j \geq 2$.

If $n_{1}^{1}=0$ we have $n_{j}^{i}=0 \forall i, \forall j$. Equation (20) becomes: $-\frac{t_{j}^{2}}{2} \boldsymbol{\alpha}-t_{j} \boldsymbol{\nu}+\Gamma_{j} \boldsymbol{b}=0_{3}$. From property 12 we conclude that it exists in general one independent vector $\boldsymbol{n} \in \mathcal{N}\left(\Xi^{\prime}\right)$ with $n_{1}^{1}=0$ only if the platform rotates around a single axis. If $n_{1}^{1} \neq 0$ we can divide equation (20) by $n_{1}^{1}$ obtaining: $-\frac{t_{j}^{2}}{2} \frac{\boldsymbol{\alpha}}{n_{1}^{1}}-t_{j} \frac{\boldsymbol{\nu}}{n_{1}^{1}}+\Gamma_{j} \frac{\boldsymbol{b}}{n_{1}^{1}}=\chi_{j}$. From property 12 we conclude that this system has in general a unique solution if the platform rotates around at least two axes and in general no solution if it rotates around a single axis or it does not rotate. Hence, we conclude that it exists in general one independent vector $\boldsymbol{n} \in \mathcal{N}\left(\Xi^{\prime}\right)$ with $n_{1}^{1} \neq 0$ only if the platform rotates around two or more axes.

In both cases (rotation around a single axis and rotation around two or more axes), the dimension of $\mathcal{N}\left(\Xi^{\prime}\right)$ is in general 1
$4.2 .3 n_{i} \geq 5$

We have the following property:

Property 15 (Biased case, $\boldsymbol{n}_{\boldsymbol{i}} \geq \mathbf{5}$ ) In the biased case, when $n_{i}=5$ and $N=1$ the Vi-SfM problem has always infinite solutions. When $n_{i} \geq 5$ and $N \geq 2$, or when $n_{i} \geq 6$ and $N=1$ the Vi-SfM problem has in general a unique solution if the platform rotates around at least two axes and two solutions if the platform rotates around a single axis.

Proof: When $n_{i}=5$ and $N=1$ the number of unknowns in (9) is 14 and the number of equations is 12. This proves the first statement. When $n_{i}=5$ and $N \geq 2$ and when $n_{i}=6, \forall N$, the number of unknowns is smaller than the number of equations.

Let us consider the case when $N \geq 2$ and $n_{i} \geq 5$. We proceed exactly as for the proof of property 14 . As in that proof, we conclude that it exists in general one independent vector $\boldsymbol{n} \in \mathcal{N}\left(\Xi^{\prime}\right)$ with $n_{1}^{1}=0$ only if the platform rotates around a single axis. On the other hand, we also conclude that in general it does not exist any vector $\boldsymbol{n} \in \mathcal{N}\left(\Xi^{\prime}\right)$ with $n_{1}^{1} \neq 0$, independently of the platform rotations. This proves the statement when $n_{i} \geq 5$ and $N \geq 2$.

It remains the case $n_{i} \geq 6, N=1$. We start by considering the case when the platform rotates around two or more axes. First of all it suffices to consider the case $n_{i}=6$. Indeed, if the dimension of $\mathcal{N}\left(\Xi^{\prime}\right)$ is zero when $n_{i}=6$, then equation (20) for $j \geq 7$ becomes: $n_{j}^{1}\left(\boldsymbol{P}_{1}^{1}+\boldsymbol{\chi}_{j}\right)=0_{3}$ which is true if and only if $n_{j}^{1}=0$ because of the assumption 1 . Let us consider the case $n_{i}=6$. From equation (20) for $j=2,3,4$, thanks to the result stated by property 12 , we know that in general we can express the vectors $\boldsymbol{\alpha}, \boldsymbol{\nu}$ and $\boldsymbol{b}$ as linear combinations of $n_{1}^{1} \boldsymbol{P}_{1}^{1}, n_{2}^{1} \boldsymbol{P}_{2}^{1}, n_{3}^{1} \boldsymbol{P}_{3}^{1}$ and $n_{4}^{1} \boldsymbol{P}_{4}^{1}$. By substituting these expressions in equation (20) for $j=5,6$ we obtain a homogeneous linear system of six equations in the six unknowns $n_{j}^{1}, 1 \leq j \leq 6$. In general, this system has full rank and therefore $n_{j}^{1}=0,1 \leq j \leq 6$.

Let us consider the case when the platform rotates around a single axis and $n_{i} \geq 6, N=1$. Thanks to property 11 we know that the dimension of $\mathcal{N}\left(\Xi^{\prime}\right)$ is at least 1. Specifically, we know that there is a non null vector in $\mathcal{N}\left(\Xi^{\prime}\right)$ whose first nine components make a vector which belongs to $\mathcal{N}\left(\Xi_{b}^{\prime \prime}\right)$. To prove that the dimension of $\mathcal{N}\left(\Xi^{\prime}\right)$ is in general 1 we proceed as in the previous case. Also in this case it suffices to consider the case $n_{i}=6$. Indeed, if the dimension of $\mathcal{N}\left(\Xi^{\prime}\right)$ is 1 when $n_{i}=6$, since the first nine components of the vector in $\mathcal{N}\left(\Xi^{\prime}\right)$ are in $\mathcal{N}\left(\Xi_{b}^{\prime \prime}\right)$, then equation (20) for $j \geq 7$ becomes as in the previous case: $n_{j}^{1}\left(\boldsymbol{P}_{1}^{1}+\boldsymbol{\chi}_{j}\right)=0_{3}$. Let us refer to the case $n_{i}=6$. This time, property 


\begin{tabular}{|c|c|}
\hline Cases & Number of Solutions \\
\hline \hline Varying Acceleration & Unique Solution \\
$n_{i}=4, N \geq 2 ; n_{i} \geq 5, \forall N$ & \\
\hline Varying Acceleration & Two Solutions \\
$n_{i}=3, N \geq 2 ; n_{i}=4, N=1$ & \\
\hline $\begin{array}{c}\text { Constant and non null Acceleration } \\
n_{i}=3, N \geq 2 ; n_{i} \geq 4, \forall N\end{array}$ & Two Solutions \\
\hline Null Acceleration & Infinite Solutions \\
$\forall n_{i}, \forall N$ & \\
\hline Any Motion & Infinite Solutions \\
$n_{i} \leq 2, \forall N ; n_{i}=3, N=1$ & \\
\hline
\end{tabular}

Table 1 Number of distinct solutions for the Vi-SfM problem in the unbiased case

\begin{tabular}{|c|c|}
\hline Cases & Number of Solutions \\
\hline \hline Rotation around 2 or more axes \\
Varying Acceleration & Unique Solution \\
$n_{i}=5, N \geq 2 ; n_{i} \geq 6, \forall N$ & \\
\hline $\begin{array}{c}\text { Rotation around a single axis } \\
\text { Varying Acceleration }\end{array}$ & Two Solutions \\
$n_{i}=5, N \geq 2 ; n_{i} \geq 6, \forall N$ & \\
\hline $\begin{array}{c}\text { Rotation around } 1 \text { or more axes } \\
\text { Varying Acceleration } \\
n_{i}=4, N \geq 2\end{array}$ & Two Solutions \\
\hline $\begin{array}{c}\text { Rotation around } 2 \text { or more axes } \\
\text { Constant and non null Acceleration } \\
n_{i}=4,5, N \geq 2 ; n_{i} \geq 6, \forall N\end{array}$ & Two Solutions \\
\hline $\begin{array}{c}\text { Rotation around a single axis } \\
\text { Constant Acceleration }\end{array}$ & Infinite Solutions \\
\hline $\begin{array}{c}\text { No rotation } \\
\forall n_{i}, \forall N\end{array}$ & Infinite Solutions \\
$\forall n_{i}, \forall N$ & Infinite Solutions \\
\hline Null Acceleration & Infinite Solutions \\
\hline$n_{i} \leq 3, \forall N ; n_{i}=4,5, N=1$ & \\
\hline
\end{tabular}

Table 2 Number of distinct solutions for the Vi-SfM problem in the biased case

12 allows us to state that we can in general obtain 8 components among the nine components of the vectors $\boldsymbol{\alpha}, \boldsymbol{\nu}$ and $\boldsymbol{b}$ as linear combinations of $n_{1}^{1} \boldsymbol{P}_{1}^{1}, n_{2}^{1} \boldsymbol{P}_{2}^{1}, n_{3}^{1} \boldsymbol{P}_{3}^{1}$ and $n_{4}^{1} \boldsymbol{P}_{4}^{1}$ and the remaining component (denoted with $w$ ) of the three vectors $\boldsymbol{\alpha}, \boldsymbol{\nu}$ and $\boldsymbol{b}$. By substituting these expressions in equation (20) for $j=5,6$ we obtain a homogeneous linear system of six equations in the seven unknowns $n_{j}^{1}, 1 \leq j \leq 6$ and $w$. In general, this system has a one dimensional null space

\section{Discussion}

\subsection{Summary of the theoretical results}

Tables 1 and 2 summarize our results by providing the number of solutions case by case, respectively in the case without bias (table 1) and with bias (table 2). Note that these tables do not account the point-features lay-

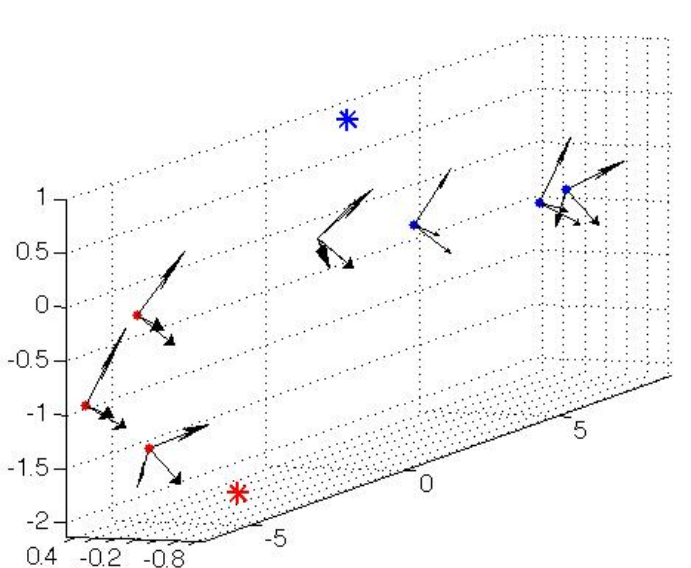

Fig. 2 Illustration of two distinct solutions for the unbiased case with $n_{i}=4, N=1$ (star symbols indicate the position of the point-feature respectively for the two solutions).

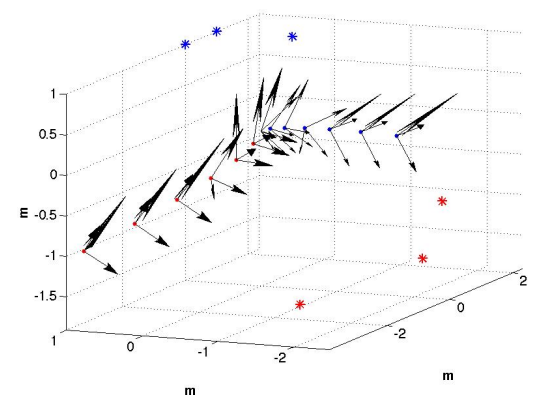

Fig. 3 Illustration of two distinct solutions for the unbiased case with constant acceleration (star symbols indicate the position of the point-features respectively for the two solutions).

out. Specifically, the motion and the point-features are not supposed to be either coplanar or collinear. Regarding these cases, necessary conditions are provided in properties 1 and 2 . In table 2 , by motion with constant acceleration we mean the special motion described in property 10.

Figures 2 and 3 illustrate two cases when the ViSfM problem has two distinct solutions. The platform configurations and the position of the point-features in the global frame are shown. The two solutions are in blue and red. The platform configuration at the initial time is the same for both the solutions and it is in black. Both figures show unbiased cases. Fig 2 regards the case of one point-feature seen in four images. Fig 3 displays the case of constant acceleration: the case of three point-features in seven images has been considered and the seven poses of the platform at the time when the images are taken are shown in the figure together with the position of the point-features. 


\subsection{Simulations}

In this section we show the benefit of using the closed solution for initializing a filter based approach to solve the Vi-SfM problem. Specifically, we generate Monte Carlo simulations according to the following four scenarios:

$S_{a}$ ideal conditions, i.e., all the measurements are noiseless, the gyroscope bias is set to zero, the accelerometer bias is constant, the extrinsic camera calibration is perfectly known;

$S_{b}$ as in $S_{a}$ but now the visual and the inertial measurements are corrupted by noise, as explained in section 5.2 .2

$S_{c}$ as in $S_{b}$ but now the gyroscopes and the accelerometers are affected by a time varying bias, as explained in section 5.2.1;

$S_{d}$ as in $S_{c}$ but now the camera extrinsic calibration is affected by an error, as explained in section 5.2.1.

In section 3 we formulated the Vi-SfM problem as the problem of determining the vectors: $\boldsymbol{P}^{i},(i=1 \ldots N)$, $\boldsymbol{V}, \boldsymbol{G}$ (and also $\boldsymbol{B}$ in presence of bias). For the sake of clarity, in this section we choose to show the results in a global frame. For this reason, we need to consider at least two point-features. Indeed, two is the minimum number of point-features to uniquely define a global frame, provided that they do not lie on the same vertical axis (defined by the gravity). We define the global frame as follows: first, we define one of the point-features as the origin of the frame. The $z$-axis coincides with the gravity axis but with opposite direction. Finally, the $x$-axis is defined by requiring that the second pointfeature belongs to the $x z$-plane. In other words, the second point-feature has zero $y$-coordinate. In these settings, the Vi-SfM can be defined as the estimation of the platform configuration and the estimation of the $x$ and the $z$ coordinate of the second point-feature (from now on, $p_{x}$ and $p_{z}$, respectively). By adding more pointfeatures, the state to be estimated also includes all the three coordinates of each point-feature. We adopt an Extended Kalman Filter $(E K F)$ to perform this estimation. The state to be estimated is:

$$
\boldsymbol{x}^{\boldsymbol{e}} \equiv\left[\boldsymbol{r}, \boldsymbol{v}, q, p_{x}, p_{z}, \boldsymbol{B}, \boldsymbol{B}_{\boldsymbol{\Omega}}, \boldsymbol{p}^{3}, \ldots, \boldsymbol{p}^{N}\right]^{T}
$$

where $q$ is a unit quaternion characterizing the platform orientation and $\boldsymbol{B}_{\boldsymbol{\Omega}}$ is the bias on the measurements provided by the gyroscopes.

By collecting the sensor measurements during the time-interval $\left[T_{i n}, T_{f i n}\right]$, the closed solution discussed in the previous sections allows us to determine the vectors $\boldsymbol{P}^{i},(i=1,2, \ldots, N), \boldsymbol{V}, \boldsymbol{G}$ and $\boldsymbol{B}$ at the time
$T_{i n}$. Note that, when $N \geq 2$, having the vectors $\boldsymbol{P}^{i}, \boldsymbol{V}$, $\boldsymbol{G}$ and $\boldsymbol{B}$ at the time $T_{i n}$, allows us to build the state $\boldsymbol{x}^{\boldsymbol{e}}$ at time $T_{i n}$ (with the exception of $\boldsymbol{B}_{\boldsymbol{\Omega}}$ ).

In this section, we investigate how the performance of the $E K F$ depends on its initialization and how this performance can be improved by using the closed solution to initialize the state. We adopt eight initialization for our filter, denoted by $I_{1}, I_{2}, \cdots, I_{8}$. In $I_{1}$ the initial state coincides with the true state. In $I_{2}, I_{3}, I_{4}, I_{5}$ the initial state coincides with the true state regarding the angular components (i.e., the roll, pitch and yaw angles), while the metric components are corrupted by changing the scale factor. In particular, the scale factor is set equal to $0.95\left(I_{2}\right), 1.1\left(I_{3}\right), 0.8\left(I_{4}\right)$ and $1.3\left(I_{5}\right)$. In $I_{6}, I_{7}$ the initial state coincides with the true state regarding all the metric components, while all the angular components are corrupted by adding an error of $1 \mathrm{deg}\left(I_{6}\right)$ and $3 \mathrm{deg}\left(I_{7}\right)$. Finally, in $I_{8}$ the initial state is obtained by using our closed form solution. In particular, the initial state is obtained by using the first 6 camera observations (i.e. by considering the time interval $\left.\left[T_{i n}=0, T_{\text {fin }}=0.5\right] \mathrm{s}\right)$. Since the closed solution does not provide the initial $\boldsymbol{B}_{\boldsymbol{\Omega}}$, its initial value will be set to zero.

\subsubsection{Simulated Trajectories}

All the trajectories are randomly generated starting from the following initial true state:

$\boldsymbol{r}\left(T_{\text {in }}\right)=[0.5,0.5,0.5] m ; \boldsymbol{v}\left(T_{\text {in }}\right)=[0.1,0.1,0.1] \mathrm{ms}^{-1}$

$q\left(T_{i n}\right)=1$, which corresponds to the platform attitude roll $=$ pitch $=$ yaw $=0 \mathrm{deg} ; \boldsymbol{B}\left(T_{i n}\right)=0.05 \hat{\boldsymbol{\mu}} \mathrm{m} \mathrm{s}^{-2}$, where $\hat{\boldsymbol{\mu}}$ is the unit vector pointing in the direction $[1,1,1] ; p_{x}=2 m$ and $p_{z}=1 m$. In the scenarios $S_{c}$ and $S_{d}$ also the gyroscope is affected by a bias, whose initial value is set as follows: $\boldsymbol{B}_{\boldsymbol{\Omega}}\left(T_{i n}\right)=0.5 \hat{\boldsymbol{\mu}} \mathrm{deg} \mathrm{s}{ }^{-1}$. Additionally, in these two scenarios both the biases are time-dependent. Specifically, they are modelled as independent random walks (for all the three components of both), whose mean values are the initial ones and their variances increase linearly with time. For the gyroscopes, the three variances are set equal to $(50 \mathrm{deg} / \mathrm{h})^{2}$ at $100 \mathrm{~s}$ and for the accelerometers are set equal to $\left(1 \mathrm{~m} / \mathrm{h}^{2}\right)^{2}$ at $100 \mathrm{~s}$ (see [25]). We assume that the camera and the IMU frame coincide (i.e., they have the same origin and the same orientation). In the last scenario $\left(S_{d}\right)$ we characterize an error in the extrinsic calibration by setting the actual position of the origin of the camera frame in the IMU frame to $[0.002$, $0.003,0.004] \mathrm{m}$ and the actual orientation $q_{c a m}=1-$ $2.310^{-5}+(3.5 i-5.2 j+2.6 k) 10^{-3}$, which corresponds 
to the attitude roll $=0.4 \mathrm{deg}$, pitch $=-0.6 \mathrm{deg}$ and yaw $=0.3 \mathrm{deg}$.

We also considered the case of more than two pointfeatures $(N \geq 3)$, obtaining similar results in terms of performance and, for the sake of brevity, in the following we only refer to the case of $N=2$.

The trajectories are generated by randomly generating the linear and angular acceleration of the platform at $100 \mathrm{~Hz}$. In particular, at each time step, the three components of the linear acceleration and the angular speed are generated as zero-mean Gaussian independent variables whose covariance matrices are equal to $\left(1 \mathrm{~ms}^{-2}\right)^{2} I_{3}$ and $\left(10 \mathrm{deg} s^{-1}\right)^{2} I_{3}$, respectively. The length of each trajectory is $6 s$.

\subsubsection{Simulated Sensors}

Starting from the accomplished trajectory, the true angular speed and the linear acceleration are computed at each time step of $0.01 s$ (respectively, at the $j^{\text {th }}$ time step, we denote them with $\boldsymbol{\Omega}_{j}^{\boldsymbol{t r} \boldsymbol{r} \boldsymbol{e}}$ and $\boldsymbol{A}_{\boldsymbol{j}}^{\text {true }}$ ). Starting from them, the IMU sensors are simulated by randomly generating the angular speed and the linear acceleration at each step according to the following: $\boldsymbol{\Omega}_{\boldsymbol{j}}=$ $N\left(\boldsymbol{\Omega}_{\boldsymbol{j}}^{\text {true }}-\boldsymbol{B}_{\boldsymbol{\Omega}}\left(t_{j}\right), P_{\Omega}\right)$ and $\boldsymbol{A}_{\boldsymbol{j}}=N\left(\boldsymbol{A}_{\boldsymbol{j}}^{\text {true }}-\boldsymbol{G}\left(t_{j}\right)\right.$ $\left.-\boldsymbol{B}\left(t_{j}\right), P_{A}\right)$, where $N(.,$.$) indicates the Normal dis-$ tribution whose first entry is the mean value and the second its covariance matrix and $P_{\Omega}$ and $P_{A}$ are the covariance matrices characterizing the accuracy of the $I M U$. In all the simulations we set both the matrices $P_{\Omega}$ and $P_{A}$ diagonal. In the first scenario $\left(S_{a}\right)$ we set both these matrices to zero. In the last three scenarios $\left(S_{b}, S_{c}, S_{d}\right)$ we set: $P_{\Omega}=\left(1 \mathrm{deg} \mathrm{s} s^{-1}\right)^{2} I_{3}$ and $P_{A}=\left(1 \mathrm{~cm} \mathrm{~s}^{-2}\right)^{2} I_{3}$.

Regarding the camera, the provided readings are generated in the following way. By knowing the true trajectory and the true camera-IMU transformation, the true bearing angles of the two point-features in the camera frame are computed. They are computed each $0.1 s$. Then, the camera readings are generated by adding to the true values zero-mean Gaussian errors whose variance is equal to $(1 \mathrm{deg})^{2}$ for all the readings (for the last three scenarios, $\left.S_{b}, S_{c}, S_{d}\right)$. In the first scenario $\left(S_{a}\right)$ the camera readings coincide with the true bearing angles.

\subsubsection{Simulation Results}

For each setting (i.e., each scenario among $S_{a}, S_{b}, S_{c}$ and $S_{d}$ and each initial state $\left.I_{1}, \cdots, I_{8}\right)$ we ran 100 simulations. For each simulation we computed the error, i.e. the difference between the true value and the one estimated. We provide the error on the platform

\begin{tabular}{|c||rcc|ccc|ccc|}
\hline Sim & \multicolumn{4}{||}{ Position $(\mathrm{cm})$} & \multicolumn{4}{|c|}{ Velocity $\left(\frac{\mathrm{cm}}{\mathrm{s}}\right)$} & \multicolumn{4}{|c|}{ Attitude $(\mathrm{deg})$} \\
\hline$S_{a}$ & 0.06 & 0.04 & 0.15 & 1.4 & 0.02 & 1.5 & 0.01 & 0.007 & 0.03 \\
\hline$S_{b}$ & 1.0 & 1.0 & 4.1 & 1.8 & 0.46 & 2.6 & 0.06 & 0.06 & 0.22 \\
\hline$S_{c}$ & 2.5 & 1.2 & 5.8 & 4.2 & 1.3 & 5.7 & 0.45 & 0.37 & 0.7 \\
\hline$S_{d}$ & 3.4 & 1.7 & 8.2 & 5.4 & 1.9 & 6.5 & 0.68 & 0.58 & 1.4 \\
\hline
\end{tabular}

Table 3 Performance of the closed form solution in determining the initial state in the four considered scenarios $\left(S_{a}, S_{b}, S_{c}, S_{d}\right)$.

position, velocity and attitude. All these errors are defined as the following scalars. The position error is the magnitude of the vector, which is the difference between the true platform position and the one estimated. Similarly, the velocity error is the magnitude of the vector, which is the difference between the true velocity and the one estimated. Finally, the attitude error is the mean value of the roll error, pitch error and yaw error. Each of these is defined as the absolute value of the difference between the true value and the one estimated. We evaluate both the performance of the closed form solution in determining the initial state and the performance of the $E K F$. Regarding the latter, the errors are averaged along the entire trajectory.

For each setting, the 100 simulations provided a distribution for all the previous errors. In tables 3 and 4 we provide three values in order to characterize each distribution: they are the mean, the standard deviation and the maximum, respectively. Table 3 shows the performance of the proposed closed form solution in determining the initial state. The position error is of few centimetres in the hardest scenarios $S_{c}, S_{d}$, which corresponds to an error on the absolute scale smaller than $3 \%$. Regarding the attitude, the error is smaller than $0.7 \mathrm{deg}$. Table 4 shows the performance of the $E K F$ in estimating the state along the entire trajectory. The best estimates are obviously the ones obtained with a perfect initialization $\left(I_{1}\right)$. On the other hand, the performance achieved by initializing the state through the proposed closed form solution $\left(I_{8}\right)$ is always much better than the one obtained in all the other cases. In particular, this performance is closer to the one obtained with a perfect initialization than to the performance achieved in the case of an initial scale equal to 0.95 and perfect attitude $\left(I_{2}\right)$ and the performance achieved in the case of perfect initial metric components and initial roll pitch and yaw affected by an error of 1 deg $\left(I_{6}\right)$.

By considering initial states corrupted by an error larger than the ones in $I_{4}, I_{5}$ and $I_{7}$ we often obtained a filter divergence. 
$S_{a}$

\begin{tabular}{|c|c|c|c|}
\hline In. & Position $(\mathrm{cm})$ & Velocity $\left(\frac{\mathrm{cm}}{\mathrm{s}}\right)$ & Attitude (deg) \\
\hline$I_{1}$ & $3 \mathrm{e}-3 \quad 2 \mathrm{e}-3 \quad 0.01$ & $5 e-3 \quad 4 e-3 \quad 0.02$ & $6-4 \quad 5 e-5 \quad 2 e-3$ \\
\hline$I_{2}$ & $\begin{array}{lll}1.4 & 0.47 & 2.8\end{array}$ & $\begin{array}{lll}1.2 & 0.64 & 3.6\end{array}$ & $\begin{array}{lll}0.13 & 0.06 & 0.28\end{array}$ \\
\hline$I_{3}$ & $\begin{array}{lll}4.1 & 1.7 & 9.8\end{array}$ & $\begin{array}{lll}4.0 & 2.8 & 9.3\end{array}$ & $\begin{array}{llll}0.45 & 0.36 & 1.8\end{array}$ \\
\hline$I_{4}$ & $\begin{array}{lll}16 & 8.9 & 37\end{array}$ & $\begin{array}{lll}19 & 9.9 & 40\end{array}$ & $\begin{array}{lll}2.5 & 1.9 & 8.7\end{array}$ \\
\hline$I_{5}$ & $\begin{array}{lll}30 & 17 & 76\end{array}$ & $\begin{array}{lll}32 & 20 & 90\end{array}$ & $\begin{array}{lll}3.4 & 2.0 & 8.6\end{array}$ \\
\hline$I_{6}$ & $\begin{array}{lll}2.8 & 3.5 & 4.7\end{array}$ & $\begin{array}{lll}4.6 & 5.6 & 13\end{array}$ & $\begin{array}{lll}0.63 & 0.66 & 2.9\end{array}$ \\
\hline$I_{7}$ & $\begin{array}{lll}20 & 15 & 78\end{array}$ & $\begin{array}{lll}35 & 41 & 233\end{array}$ & $\begin{array}{lll}3.5 & 3.7 & 11\end{array}$ \\
\hline$I_{8}$ & $\begin{array}{lll}0.03 & 0.01 & 0.06\end{array}$ & $\begin{array}{ccc}0.05 & 0.02 & 0.09\end{array}$ & $\begin{array}{lll}0.01 & 4 \mathrm{e}-3 & 0.02\end{array}$ \\
\hline
\end{tabular}

\begin{tabular}{|c|c|c|c|}
\hline \multicolumn{4}{|c|}{$S_{b}$} \\
\hline In. & Position $(\mathrm{cm})$ & Velocity $\left(\frac{\mathrm{cm}}{\mathrm{s}}\right)$ & Attitude $(\mathrm{deg})$ \\
\hline$I_{1}$ & $\begin{array}{lll}2.0 & 1.0 & 4.1\end{array}$ & $\begin{array}{lll}2.7 & 1.2 & 5.9\end{array}$ & $\begin{array}{lll}0.35 & 0.13 & 0.63\end{array}$ \\
\hline$I_{2}$ & $\begin{array}{lll}3.0 & 1.4 & 6.0\end{array}$ & $\begin{array}{lll}3.4 & 1.3 & 6.2 \\
\end{array}$ & $\begin{array}{lll}0.48 & 0.33 & 1.8\end{array}$ \\
\hline$I_{3}$ & $\begin{array}{lll}5.2 & 2.6 & 11\end{array}$ & $\begin{array}{lll}4.8 & 3.1 & 15\end{array}$ & $\begin{array}{lll}0.74 & 0.48 & 2.1\end{array}$ \\
\hline$I_{4}$ & $\begin{array}{lll}18 & 17 & 96\end{array}$ & $\begin{array}{lll}22 & 22 & 127\end{array}$ & $\begin{array}{lll}3.0 & 3.9 & 21\end{array}$ \\
\hline$I_{5}$ & $\begin{array}{lll}39 & 46 & 262\end{array}$ & $\begin{array}{lll}44 & 62 & 357\end{array}$ & $\begin{array}{lll}4.1 & 3.1 & 13\end{array}$ \\
\hline$I_{6}$ & $\begin{array}{lll}3.3 & 1.6 & 6.7\end{array}$ & $\begin{array}{lll}5.1 & 6.1 & 9.7\end{array}$ & $\begin{array}{lll}0.73 & 0.34 & 2.7\end{array}$ \\
\hline$I_{7}$ & $\begin{array}{lll}26 & 38 & 213\end{array}$ & $\begin{array}{lll}44 & 72 & 393\end{array}$ & $\begin{array}{lll}4.4 & 3.3 & 14\end{array}$ \\
\hline$I_{8}$ & $\begin{array}{lll}2.2 & 1.2 & 5.0\end{array}$ & $\begin{array}{lll}3.1 & 1.4 & 6.9 \\
\end{array}$ & $\begin{array}{lll}0.38 & 0.18 & 1.0\end{array}$ \\
\hline
\end{tabular}

\begin{tabular}{|c|c|c|c|}
\hline \multicolumn{4}{|c|}{$S_{c}$} \\
\hline In. & Position $(\mathrm{cm})$ & Velocity $\left(\frac{\mathrm{cm}}{\mathrm{s}}\right)$ & Attitude (deg) \\
\hline$I_{1}$ & $\begin{array}{lll}2.3 & 1.1 & 4.9 \\
\end{array}$ & $\begin{array}{lll}3.8 & 1.3 & 7.0 \\
\end{array}$ & $\begin{array}{lll}0.37 & 0.18 & 1.0\end{array}$ \\
\hline$I_{2}$ & $\begin{array}{lll}3.3 & 1.4 & 6.7 \\
\end{array}$ & $\begin{array}{lll}5.4 & 1.4 & 8.2 \\
\end{array}$ & $\begin{array}{lll}0.59 & 0.34 & 2.1 \\
\end{array}$ \\
\hline$I_{3}$ & $\begin{array}{lll}5.5 & 2.7 & 13\end{array}$ & $\begin{array}{lll}5.6 & 2.9 & 17\end{array}$ & $\begin{array}{lll}0.83 & 0.48 & 2.8 \\
\end{array}$ \\
\hline$I_{4}$ & $\begin{array}{lll}23 & 42 & 236\end{array}$ & $\begin{array}{lll}34 & 78 & 433\end{array}$ & $\begin{array}{lll}3.6 & 6.9 & 38\end{array}$ \\
\hline$I_{5}$ & $\begin{array}{lll}37 & 39 & 218\end{array}$ & $\begin{array}{lll}49 & 91 & 500\end{array}$ & $\begin{array}{lll}4.0 & 3.9 & 20\end{array}$ \\
\hline$I_{6}$ & $\begin{array}{lll}4.2 & 4.6 & 7.7 \\
\end{array}$ & $\begin{array}{lll}6.0 & 5.1 & 11\end{array}$ & $\begin{array}{lll}0.92 & 0.74 & 3.7\end{array}$ \\
\hline$\overline{I_{7}}$ & $\begin{array}{lll}71 & 112 & 145\end{array}$ & $\begin{array}{lll}107 & 384 & 205\end{array}$ & $\begin{array}{lll}5.7 & 7.4 & 38\end{array}$ \\
\hline$I_{8}$ & $\begin{array}{lll}2.7 & 1.8 & 4.5\end{array}$ & $\begin{array}{lll}4.4 & 1.7 & 9.7\end{array}$ & $\begin{array}{lll}0.50 & 0.26 & 1.4\end{array}$ \\
\hline
\end{tabular}

\begin{tabular}{|c|c|c|c|}
\hline In. & Position $(\mathrm{cm})$ & Velocity $\left(\frac{\mathrm{cm}}{\mathrm{s}}\right)$ & Attitude (deg) \\
\hline$I_{1}$ & $\begin{array}{lll}3.3 & 1.9 & 8.4\end{array}$ & $\begin{array}{lll}4.4 & 2.5 & 14\end{array}$ & $\begin{array}{lll}0.68 & 0.50 & 2.1\end{array}$ \\
\hline$I_{2}$ & $\begin{array}{lll}4.1 & 2.0 & 9.2\end{array}$ & $\begin{array}{lll}7.3 & 2.6 & 12\end{array}$ & $\begin{array}{lll}0.84 & 0.79 & 3.5\end{array}$ \\
\hline$I_{3}$ & $\begin{array}{lll}9.3 & 8.9 & 18\end{array}$ & $\begin{array}{lll}11 & 13 & 27\end{array}$ & $\begin{array}{lll}1.7 & 1.8 & 4.7\end{array}$ \\
\hline$I_{4}$ & $\begin{array}{lll}38 & 114 & 617\end{array}$ & $\begin{array}{lll}57 & 186 & 800\end{array}$ & $\begin{array}{lll}4.0 & 7.0 & 38\end{array}$ \\
\hline$I_{5}$ & $\begin{array}{lll}103 & 376 & 201\end{array}$ & $\begin{array}{lll}156 & 635 & 339\end{array}$ & $\begin{array}{lll}5.7 & 10 & 55\end{array}$ \\
\hline$I_{6}$ & $\begin{array}{lll}4.5 & 3.2 & 10\end{array}$ & $\begin{array}{lll}6.9 & 5.5 & 13\end{array}$ & $\begin{array}{lll}0.94 & 0.59 & 4.2\end{array}$ \\
\hline$I_{7}$ & $\begin{array}{lll}85 & 95 & 137\end{array}$ & $119 \quad 134 \quad 271$ & $\begin{array}{lll}6.8 & 8.7 & 48\end{array}$ \\
\hline$I_{8}$ & $\begin{array}{lll}3.8 & 1.8 & 8.5\end{array}$ & $\begin{array}{lll}6.8 & 1.9 & 11\end{array}$ & $\begin{array}{lll}0.76 & 0.43 & 1.9\end{array}$ \\
\hline
\end{tabular}

Table 4 Performance of the $E K F$ for the four considered scenarios $\left(S_{a}, S_{b}, S_{c}, S_{d}\right)$ and for the eight considered initial conditions $\left(I_{1}, \cdots, I_{8}\right) . I_{8}$ corresponds to the initialization obtained by the proposed closed form solution.

\section{Conclusion}

In this paper we derived a simple and intuitive closed solution to the visual-inertial structure from motion problem. We used this derivation to investigate the intrinsic properties of the Vi-SfM problem and to identify the conditions under which the problem can be solved in closed form. In particular, we showed that the problem can have a unique solution or two distinct solutions or infinite solutions depending on the trajectory, on the number of point-features and their layout and on the number of monocular images where the same point-features are seen. The investigation was also per- formed in the case when the inertial data are biased, showing that, in this latter case, more images and more restrictive conditions on the trajectory are required in order to have a finite number of solutions.

The most useful applications of the closed-form solution here derived will be in all the applicative domains which need to solve the SfM problem with low-cost sensors and which do not demand any infrastructure (e.g., in GPS denied environment). Additionally, our results could also play an important role in the framework of neuroscience. Indeed, our findings show that it is possible to easily distinguish linear acceleration from gravity. Specifically, the closed form solution performs this determination by a very simple matrix inversion. This problem has been investigated in neuroscience [18]. Our results could provide a new insight to this mechanism since they clearly characterize the conditions (type of motion, features layout) under which this determination can be performed.

\section{References}

1. L. Armesto, J. Tornero, and M. Vincze Fast Ego-motion Estimation with Multi-rate Fusion of Inertial and Vision, The International Journal of Robotics Research 2007 26: 577-589

2. A. Berthoz, B. Pavard and L.R. Young, Perception of Linear Horizontal Self-Motion Induced by Peripheral Vision (Linearvection) Basic Characteristics and Visual-Vestibular Interactions, Exp. Brain Res. 23, 471-489 (1975).

3. M. Bryson and S. Sukkarieh, Observability Analysis and Active Control for Airbone SLAM, IEEE Transaction on Aerospace and Electronic Systems, vol. 44, no. 1, 261-280, 2008

4. Alessandro Chiuso, Paolo Favaro, Hailin Jin and Stefano Soatto, "Structure from Motion Causally Integrated Over Time", IEEE Transactions on Pattern Analysis and Machine Intelligence, 24(4), pp 523-535, 2002

5. Andrew J. Davison, Ian D. Reid, Nicholas D.Molton and Olivier Stasse, "MonoSLAM: Real-Time Single Camera SLAM", IEEE Transactions on Pattern Analysis and Machine Intelligence, 29(6), pp 1052-1067, 2007

6. Dokka K., MacNeilage P. R., De Angelis G. C. and Angelaki D. E., Estimating distance during self-motion: a role for visual-vestibular interactions, Journal of Vision (2011) 11(13):2, 1-16

7. T.C Dong-Si, A.I. Mourikis, Initialization in Vision-aided Inertial Navigation with Unknown Camera-IMU Calibration," Proceedings of the IEEE/RSJ International Conference on Intelligent Robots and Systems (IROS), Vilamoura, Portugal, October 7-12 2012, pp. 1064-1071.

8. J. A. Farrell, Aided Navigation: GPS and High Rate Sensors. McGraw- Hill, 2008.

9. C. R. Fetsch, G. C. DeAngelis and D. E. Angelaki, Visualvestibular cue integration for heading perception: Applications of optimal cue integration theory, Eur J Neurosci. 2010 May ; 31(10): 1721-1729

10. P. Gemeiner, P. Einramhof, and M. Vincze, Simultaneous Motion and Structure Estimation by Fusion of Inertial and Vision Data, The International Journal of Robotics Research 2007 26: 591-605 
11. Richard I. Hartley (June 1997). "In Defense of the EightPoint Algorithm". IEEE Transaction on Pattern Recognition and Machine Intelligence 19 (6): 580-593.

12. B. R. Hunt, T. Sauer and J. A. Yorke, Prevalence: a translation-invariant "almost every" on infinitedimensional spaces, BULLETIN OF THE AMERICAN MATHEMATICAL SOCIETY Volume 27, Number 2, October 1992

13. E. Jones and S. Soatto, "Visual-inertial navigation, mapping and localization: A scalable real-time causal approach", The International Journal of Robotics Research, vol. 30, no. 4, pp. 407-430, Apr. 2011.

14. J. Kelly and G. Sukhatme, Visual-inertial simultaneous localization, mapping and sensor-to-sensor self-calibration, Int. Journal of Robotics Research, vol. 30, no. 1, pp. 56-79, 2011.

15. Kim, J. and Sukkarieh, S. Real-time implementation of airborne inertial-SLAM, Robotics and Autonomous Systems, 2007, 55, 62-71

16. H. Christopher Longuet-Higgins (September 1981). "A computer algorithm for reconstructing a scene from two projections". Nature 293: 133-135.

17. A. Martinelli, Vision and IMU Data Fusion: Closed-Form Solutions for Attitude, Speed, Absolute Scale and Bias Determination, Transaction on Robotics, Volume 28 (2012), Issue 1 (February), pp 44-60.

18. Merfeld D. M., Zupan L. and Peterka R. J., Humans use internal models to estimate gravity and linear acceleration, Nature, 398, pp 615-618, 1999

19. C. D. Meyer, Matrix Analysis and Applied Linear Algebra, SIAM, 2000

20. D. Nistér, An efficient solution to the five-point relative pose problem, IEEE Transactions on Pattern Analysis and Machine Intelligence (PAMI), 26(6):756-770, June 2004

21. D. Strelow and S. Singh, Motion estimation from image and inertial measurements, International Journal of Robotics Research, 23(12), 2004

22. M. Veth, and J. Raquet, Fusing low-cost image and inertial sensors for passive navigation, Journal of the Institute of Navigation, vol. 54(1), 2007

23. Weiss., S., Scaramuzza, D., Siegwart, R., MonocularSLAM-Based Navigation for Autonomous Micro Helicopters in GPS-Denied Environments, Journal of Field Robotics, Volume 28, issue 6, 2011

24. Weiss., S., Vision Based Navigation for Micro Helicopters, PhD thesis, Diss. ETH No. 20305

25. Woodman, Oliver J., An introduction to inertial navigation, Technical Report, University of Cambridge, Computer Laboratory, 2007, UCAM-CL-TR-696 